\title{
Stochastic Dominance Statistics for Risk Averters and Risk Seekers: An Analysis of Stock Preferences for USA and China
}

\author{
Zhidong Bai \\ KLASMOE and School of Mathematics and Statistics \\ Northeast Normal University \\ Department of Statistics and Applied Probability \\ and Risk Management Institute \\ National University of Singapore \\ Hua $\mathrm{Li}$ \\ Department of Statistics and Applied Probability \\ National University of Singapore \\ Michael McAleer \\ Econometric Institute, Erasmus School of Economics, Erasmus \\ University Rotterdam \\ Tinbergen Institute, The Netherlands \\ Department of Quantitative Economics, Complutense University of \\ Madrid \\ Institute of Economic Research, Kyoto University \\ Wing-Keung Wong \\ Department of Economics \\ Hong Kong Baptist University
}

June 6, 2012

Corresponding author: Wing-Keung Wong, Department of Economics, Hong Kong Baptist University, Kowloon Tong, Hong Kong. Tel: (852)-3411-7542, Fax: (852)-3411-5580, Email: awong@hkbu.edu.hk 


\section{Acknowledgments}

This research is partially supported by Northeast Normal University, National University of Singapore, Research Grants Council of Hong Kong, and Hong Kong Baptist University. The third author wishes to acknowledge the financial support of the Australian Research Council, National Science Council, Taiwan, and the Japan Society for the Promotion of Science. The fourth author would like to thank Professors Robert B. Miller and Howard E. Thompson for their continuous guidance and encouragement. 


\title{
Stochastic Dominance Statistics for Risk Averters and Risk Seekers: An Analysis of Stock Preferences for USA and China
}

\begin{abstract}
We derive the limiting process of the stochastic dominance statistics for risk averters as well as for risk seekers when the underlying processes might be dependent or independent. We take account of the dependency of the partitions and propose a bootstrap method to decide the critical point. In addition, we illustrate the applicability of the stochastic dominance statistics for both risk averters and risk seekers to analyze the dominance relationship between the Chinese and US stock markets in the entire period as well as the sub-periods before and after the financial crises, including the internet bubble and the recent sub-prime crisis. The findings could be used to draw inferences on the preferences of risk averters and risk seekers in investing in the Chinese and US stock markets. The results also enable us to examine whether there is any arbitrage opportunity in these markets and whether these markets are efficient and investors are rational.
\end{abstract}

Keywords: Stochastic dominance, risk aversion, risk seeking, test statistic, hypothesis testing.

JEL Classification: $\quad$ C12, G1, G12, G15 


\section{Introduction}

The stochastic dominance (SD) tests have been developing to test for statistically significant differences between any two cumulative density functions, say $F$ and $G$, of two assets, $Y$ and $Z$, drawing inference for the preferences for risk averters. Davidson and Duclos (hereafter DD, 2000) develop the SD statistic, $T_{j}(x)(j=1 ; 2 ; 3)$, to determine the levels of significance for the first-, second-, and third-order SD for two distributions $F$ and $G$. The DD test compares distributions at a finite number of grid points, and under the null hypothesis of equality of $F$ and $G$, Davidson and Duclos show that $T_{j}$ is asymptotically distributed as the studentized maximum modulus (SMM) distribution. Barrett and Donald (2003) comment that too few grids will miss information about the distributions between any two consecutive grids whereas, according to Richmond (1982), one may argue that too many grids will violate the independence assumption required by the SMM distribution. Fong et al. (2005) and others suggest using 10 major partitions with 10 minor partitions within any two consecutive major partitions in each comparison. We note that the limiting distributions of $T_{j}(x)$ are not independent even for two points of partition. More importantly, the limiting correlations would depend on the null distribution $F=G$. To solve this problem, in this paper we modify the DD statistic by taking care of the dependency of the partitions. We suggest investigating the limiting distribution of $T_{j}(x) ; a<x<b$, regarded as a stochastic process. We derive the limiting process of the DD test statistic $T_{j}(x), j=1 ; 2 ; 3$, and hence, the DD test can be performed by $\sup _{x}\left|T_{j}(x)\right|$.

It is well known that investors may not be risk-averse as they could be risk-seeking (see Meyer (1977), Stoyan (1983), Wong (2007) and the references therein for properties of the behavior of risk seekers, and investors with convex utility functions). In this paper, we also develop SD statistics to test for statistically significant differences between any two density 
functions of two assets, $Y$ and $Z$, drawing inference for the preferences for risk seekers. In addition, in many real data analyses, the random variables $Y$ and $Z$ being examined may not be independent. To take care of both dependent and independent situations, in this paper we develop the modified DD tests to cover the dependent and independent situations and provide the limiting correlations on the null distribution $F=G$. We note that Bai et al. (2012) have extended the DD test to SD test for investors with S-shaped and reverse S-shaped utility functions, our paper extend their work in the sense that we (1) compliment their work and the work by Davidson and Duclos (2000) to develop SD tests for risk averters and risk seekers, and (2) take care of the dependent and independent situations. In this paper we also propose a bootstrap method to decide the critical point of the modified DD tests so that their critical values will be closer to the true critical values.

Thereafter, we illustrate the applicability of the SD statistics for both risk averters and risk seekers analyze the dominance relationship between the Chinese and US stock markets in the entire period as well as the sub-periods before and after the financial crises, including the internet bubble and the recent sub-prime crisis. The findings could be used to draw inferences on the preferences of risk averters and risk seekers in investing in the Chinese and US stock markets. In addition, the results enable us to examine whether there is any arbitrage opportunity in these markets and whether these markets are efficient and investors are rational. We also note that a hierarchical relationship exists in SD mathematically (Sriboonchitta et al., 2009) but, in our analysis, we observe that the SD hierarchical relationship may not exist statistically. We will discuss this issue in our illustration section and propose an inference to this new finding in the literature (see, for example, Wong and Chan (2008), Broll et al. (2010), Egozcue et al. (2011), and the references therein for further information). 
The remainder of the paper is organized as follows: Section 2 introduces definitions and notation, and discusses some basic SD properties. We will develop the SD tests for risk averters and risk seekers in Section 3. In Section 4, we discuss the bootstrap method to decide the critical points of the tests. Section 5 illustrates the applicability of our proposed SD test statistics to study the preferences of risk averters and risk seekers for their investment in the Chinese and USA stock markets. Section 6 concludes our findings.

\section{Definitions, Notations, and Basic Properties}

Let $\overline{\mathbb{R}}$ be the set of extended real numbers and $\Omega=[a, b]$ be a subset of $\overline{\mathbb{R}}$. Let $\mathbb{B}$ be the Borel $\sigma$-field of $\Omega$ and $\mu$ be a measure on $(\Omega, \mathbb{B})$. We first define the functions $F^{A}$ and $F^{D}$ of the measure $\mu$ on the support $\Omega$ as $F^{A}(x)=F_{1}^{A}(x)=\mu(a, x]$ and $F^{D}(x)=F_{1}^{D}(x)=\mu[x, b)$ for all $x \in \Omega$. Function $F$ is a cumulative distribution function (CDF) and $\mu$ is a probability measure if $\mu(\Omega)=1$. From the basic probability theory, for any random variable $X$ with an associated probability measure $P$, there exists a unique induced probability measure $\mu$ on $(\Omega, \mathbb{B})$ and a CDF $F$ such that $\mu(B)=P\left(X^{-1}(B)\right)=P(X \in B)$ for any $B \in \mathbb{B}$. An integral written in the form of $\int_{A} f(t) d \mu(t)$ or $\int_{A} f(t) d F(t)$ is understood as a LebesgueStieltjes integral for integrable function $f(t)$. If the integral has the same value for all $A$ among $(c, d],[c, d)$ or $[c, d]$, we use the notation $\int_{c}^{d} f(t) d \mu(t)$ instead. In addition, if $\mu$ is a Borel measure with $\mu(c, d]=d-c$, we write the integral as $\int_{c}^{d} f(t) d t$. Random variables, denoted by $Y$ and $Z$, defined on $\Omega$, are considered together with their corresponding CDFs $F$ and $G$, respectively. The following notations will be used throughout this paper:

$$
\begin{aligned}
& \mu_{F}=\mu_{Y}=E(Y)=\int_{a}^{b} x d F(x), \quad \mu_{G}=\mu_{Z}=E(Z)=\int_{a}^{b} x d G(x) ; \text { and } \\
& H_{j}^{A}(x)=\int_{a}^{x} H_{j-1}^{A}(y) d y, \quad H_{j}^{D}(x)=\int_{a}^{x} H_{j-1}^{D}(y) d y, \quad j=2,3 ;
\end{aligned}
$$


where $H=F$ or $G .{ }^{1}$ In $(2.1), \mu_{F}=\mu_{Y}$ is the mean of $Y$, whereas $\mu_{G}=\mu_{Z}$ is the mean of $Z$.

We note that the definition of $H_{j}^{A}$ in (2.1) can be used to develop the SD theory for risk averters (see, for example, Quirk and Saposnik, 1962; Fishburn, 1964) and thus we call this type of SD ascending stochastic dominance (ASD) and call $H_{j}^{A}$ the $j^{\text {th }}$ order ASD integral or the $j^{\text {th }}$ order cumulative probability, since $H_{j}^{A}$ is integrated from $H_{j-1}^{A}$ in ascending order from the leftmost point of downside risk. On the other hand, $H_{j}^{D}$ can be used to develop the SD theory for risk seekers (see, for example, Meyer, 1977; Stoyan, 1983; Li and Wong, 1999) and thus we call this type of SD descending stochastic dominance (DSD) and call $H_{j}^{D}$ the $j^{\text {th }}$ order DSD integral or the $j^{\text {th }}$ order reversed cumulative probability, since $H_{j}^{D}$ is integrated $H_{j-1}^{D}$ in descending order from the rightmost point of upside profit. Typically, risk averters prefer assets that have a smaller probability of loss whereas risk seekers prefer assets that have a higher probability of gain, especially in upside profit. To make a choice between two assets $F$ and $G$, risk averters will compare their corresponding $j^{\text {th }}$ order ASD in integrals $F_{j}^{A}$ and $G_{j}^{A}$ and choose $F$ if $F_{j}^{A}$ is smaller, since it has a smaller probability of loss. On the other hand, risk seekers will compare their corresponding $j^{\text {th }}$ order DSD integrals $F_{j}^{D}$ and $G_{j}^{D}$ and choose $F$ if $F_{j}^{D}$ is bigger, since it has a higher probability of gain. We first state the definitions of SD for risk averters and risk seekers as follows:

Definition 2.1 Given two random variables $Y$ and $Z$ with $F$ and $G$ as their respective CDFs, $Y$ dominates $Z$ and $F$ dominates $G$ in the sense of FASD (SASD, TASD), denoted by $Y \succeq_{1}^{A} Z$ or $F \succeq_{1}^{A} G\left(Y \succeq_{2}^{A} Z\right.$ or $F \succeq_{2}^{A} G, Y \succeq_{3}^{A} Z$ or $\left.F \succeq_{3}^{A} G\right)$, if and only if $F_{1}^{A}(x) \leq$ $G_{1}^{A}(x)\left(F_{2}^{A}(x) \leq G_{2}^{A}(x), F_{3}^{A}(x) \leq G_{3}^{A}(x)\right)$ for each $x$ in $[a, b]$, where $F A S D, S A S D$, and TASD stand for first-, second-, and third-order ASD, respectively. In addition, if there

\footnotetext{
${ }^{1}$ see, for example, Wong and Li (1999), Anderson (2004), and Wong and Chan (2008).
} 
exists strict inequality for any $x$ in $[a, b]$, we say that $Y$ dominates $Z$ and $F$ dominates $G$ in the sense of SFASD, SSASD, and STASD, denoted by $Y \succ_{1}^{A} Z$ or $F \succ_{1}^{A} G, Y \succ_{2}^{A} Z$ or $F \succ_{2}^{A} G$, and $Y \succ_{3}^{A} Z$ or $F \succ_{3}^{A} G$, respectively, where SFASD, SSASD, and STASD stand for strictly first-, second-, and third-order SD, respectively.

Definition 2.2 Given two random variables $Y$ and $Z$ with $F$ and $G$ as their respective $C D F s, Y$ dominates $Z$ and $F$ dominates $G$ in the sense of FDSD (SDSD, TDSD), denoted by $Y \succeq_{1}^{D} Z$ or $F \succeq_{1}^{D} G\left(Y \succeq_{2}^{D} Z\right.$ or $F \succeq_{2}^{D} G, Y \succeq_{3}^{D} Z$ or $\left.F \succeq_{3}^{D} G\right)$, if and only if $F_{1}^{D}(x) \geq G_{1}^{D}(x)\left(F_{2}^{D}(x) \geq G_{2}^{D}(x), F_{3}^{D}(x) \geq G_{3}^{D}(x)\right)$ for each $x$ in $[a, b]$, where FDSD, SDSD, and TDSD stand for first-, second-, and third-order DSD, respectively. In addition, if there exists strict inequality for any $x$ in $[a, b]$, we say that $Y$ dominates $Z$ and $F$ dominates $G$ in the sense of SFDSD, SSDSD, and STDSD, denoted by $Y \succ_{1}^{A} Z$ or $F \succ_{1}^{A}$ $G, Y \succ_{2}^{A} Z$ or $F \succ_{2}^{A} G$, and $Y \succ_{3}^{A} Z$ or $F \succ_{3}^{A} G$, respectively, where SFDSD, SSDSD, and STDSD stand for strictly first-, second-, and third-order SD, respectively.

The SD theory is related to utility maximization. To discuss the issue, we first state the definition of utility functions for risk averters and risk seekers as follows:

Definition 2.3 For $j=1,2,3, U_{j}^{A}, U_{j}^{D}, U_{j}^{S A}$ and $U_{j}^{S D}$ are the sets of the utility functions ${ }^{2}$ such that

$$
\begin{aligned}
U_{j}^{A}\left(U_{j}^{S A}\right) & =\left\{u:(-1)^{i} u^{(i)} \leq(<) 0, i=1, \cdots, j\right\} ; \\
U_{j}^{D}\left(U_{j}^{S D}\right) & =\left\{u: u^{(i)} \geq(>) 0, i=1, \cdots, j\right\} ;
\end{aligned}
$$

where $u^{(i)}$ is the $i^{\text {th }}$ derivative of $u$.

We note that investors in $U_{j}^{A}$ are risk averse, while investors in $U_{j}^{D}$ are risk seeking. Choosing between $F$ and $G$ in accordance with a consistent set of preferences will

\footnotetext{
${ }^{2}$ We note that the theory can be easily extended to satisfy utilities defined to be non-differentiable and/or non-expected utility functions (Wong and Ma, 2008; Sriboonchitta, et al., 2009).
} 
satisfy the von Neumann-Morgenstern (1944) consistency properties. Accordingly, F is (strictly) preferred to $G$, or equivalently, $Y$ is (strictly) preferred to $Z$ if $\Delta E u \equiv$ $u(F)-u(G) \equiv u(Y)-u(Z) \geq 0(>0)$, where $u(F) \equiv u(Y) \equiv \int_{a}^{b} u(x) d F(x)$ and $u(G) \equiv u(Z) \equiv \int_{a}^{b} u(x) d G(x)$. The ASD and DSD approaches are regarded as two of the most useful tools for ranking uncertain investment prospects, since ranking assets have been proven ( $\mathrm{Li}$ and Wong, 1999) to be equivalent to utility maximization for the preferences of risk-averse and risk-seeking investors as shown in the following proposition:

Proposition 2.1 Let $Y$ and $Z$ be random variables with $C D F s$ and $G$, respectively. Suppose $u$ is a utility function. For $j=1,2$ and $3, F \succeq_{j}^{A}\left(\succ_{j}^{A}\right) G$ if and only if $u(F) \geq$ $(>) u(G)$ for any $u$ in $U_{j}^{A}\left(U_{j}^{S A}\right)$, and $F \succeq_{j}^{D}\left(\succ_{j}^{D}\right) G$ if and only if $u(F) \geq(>) u(G)$ for any $u$ in $U_{j}^{D}\left(U_{j}^{S D}\right)$.

The existence of ASD implies that the expected utility of the risk-averse investor is always higher when holding the dominant asset than when holding the dominated asset, and consequently, the dominated asset would not be chosen. We note that a hierarchical relationship exists in ASD: FASD implies SASD, which, in turn, implies TASD. However, the converse is not true. Similarly, the hierarchical relationships also exist in DSD. Thus, it is a common practice to report only the lowest dominance order of ASD and DSD.

\section{SD Tests for Risk Averters and Risk Seekers}

The tests developed by Davidson and Duclos (DD, 2000), Barrett and Donald (BD, 2003) and Linton, et al. (LMW, 2005) are the most commonly used statistics to investigate the preference for risk averters. Since the test developed by DD is found ${ }^{3}$ to be one of the most powerful statistics to test the significance of stochastic dominance and yet one of the least conservative in size, in this paper we discuss only the DD test and extend the

\footnotetext{
${ }^{3}$ See, for example, Wei and Zhang (2003), Tse and Zhang (2004) and Lean, et al. (2008) for more information.
} 
theory by modifying only the DD test for risk averters and risk seekers in the following subsections. One could easily use the idea in our paper to extend the BD and LMW tests or other SD tests to test for the preferences for risk seekers.

\subsection{ASD Tests for Risk Averters}

In light of the definition of ASD's, one may want to test the following hypothesis, for $j=1,2,3$,

$$
H_{0}^{A}: F_{j}^{A} \equiv G_{j}^{A}
$$

against three alternatives

$$
\begin{array}{ll}
H_{1}^{A}: & F \not_{j}^{A} G, \text { two sided test, } \\
H_{1 l}^{A}: & F \succ_{j}^{A} G_{j}, \text { one sided test, } \\
H_{1 r}^{A}: & F \prec_{j}^{A} G, \text { one sided test. }
\end{array}
$$

The three hypotheses are equivalent to

$H_{1}^{A}: \quad F_{j}^{A}(x) \neq G_{j}^{A}(x)$, for some $x$,

$H_{1 l}^{A}: \quad F_{j}^{A}(x) \leq G_{j}^{A}(x), \forall x$ and the inequality is strict for at least one $x$,

$H_{1 r}^{A}: \quad F_{j}^{A}(x) \geq G_{j}^{A}(x), \forall x$ and the inequality is strict for at least one $x$.

Now, assume the data $\left\{f_{i}\right\}\left(i=1,2, \cdots, N_{f}\right)$ and $\left\{g_{i}\right\}\left(i=1,2, \cdots, N_{g}\right)$ are observations drawn from the independent random variables $Y$ and $Z$ with distribution functions $F$ and $G$, respectively. The integrals $F_{j}^{A}$ and $G_{j}^{A}$ for $F$ and $G$ are defined in (2.1) for $j=1,2$ and 3. For a grid of pre-selected points $\left\{x_{k}, k=1, \cdots, K\right\}$, Davidson and Duclos propose to use the following $j^{t h}$ order ascending DD test statistic, $T_{j}^{A}(x)(j=1,2$ and 3$)$ to test for $H_{1}^{A}, H_{1 l}^{A}$, and $H_{1 r}^{A}$ :

$$
T_{j}^{A}(x)=\frac{\hat{F_{j}^{A}}(x)-\hat{G}_{j}^{A}(x)}{\sqrt{\hat{V}_{j}^{A}(x)}},
$$


where

$$
\begin{aligned}
\hat{V}_{j}^{A}(x) & =\hat{V}_{F_{j}}^{A}(x)+\hat{V}_{G_{j}}^{A}(x), \\
\hat{H}_{j}^{A}(x) & =\frac{1}{N_{h}(j-1) !} \sum_{i=1}^{N_{h}}\left(x-h_{i}\right)_{+}^{j-1}, \\
\hat{V}_{H_{j}}^{A}(x) & =\frac{1}{N_{h}}\left[\frac{1}{N_{h}((j-1) !)^{2}} \sum_{i=1}^{N_{h}}\left(x-h_{i}\right)_{+}^{2(j-1)}-\hat{H}_{j}^{A}(x)^{2}\right], H=F, G ; h=f, g .
\end{aligned}
$$

It is not difficult to show that under the null hypothesis $H_{0}^{A}$, for each fixed $x \in$ $(a, b), T_{j}^{A}(x) \rightarrow N(0,1)$, the standard normal distribution. However, the limiting joint distribution of $\left(T_{j}^{A}\left(x_{1}\right), T_{j}^{A}\left(x_{2}\right)\right)$ does not have independent components for any $x_{1} \neq x_{2}$. More importantly, the limiting correlation depends on the null in which the distribution functions $F=G$. Therefore, it is empirically difficult to test the hypotheses described in (3.1). One may incorporate the idea proposed by Bishop, et al. (1992) to test the null hypothesis for a pre-designated finite number of values $\left\{x_{k}, k=1, \cdots, K\right\}$ and then test the following weaker hypotheses:

$$
\begin{aligned}
& H_{0}^{A K}: F_{j}^{A}\left(x_{k}\right)=G_{j}^{A}\left(x_{k}\right) \text { for all } x_{k} ; \\
& H_{1}^{A K}: F_{j}^{A}\left(x_{k}\right) \neq G_{j}^{A}\left(x_{k}\right) \text { for some } x_{k} \\
& H_{1 l}^{A K}: F_{j}^{A}\left(x_{k}\right) \leq G_{j}^{A}\left(x_{k}\right) \text { for all } x_{k}, F_{j}^{A}\left(x_{k}\right)<G_{j}^{A}\left(x_{k}\right) \text { for some } x_{k} ; \\
& H_{1 r}^{A K}: F_{j}^{A}\left(x_{k}\right) \geq G_{j}^{A}\left(x_{k}\right) \text { for all } x_{k} F_{j}^{A}\left(x_{k}\right)>G_{j}^{A}\left(x_{k}\right) \text { for some } x_{k} .
\end{aligned}
$$

Under the null hypothesis $H_{0}^{A K}$, DD show that $T_{j}^{A}$ is asymptotically distributed as the studentized maximum modulus (SMM) distribution (Richmond, 1982). To implement the DD test, the $T_{j}^{A}$ is computed at each grid point and the null hypothesis, $H_{0}^{A K}$, is rejected if

$\max _{k \leq K}\left|T_{j}^{A}\left(x_{k}\right)\right|>M_{\infty, \alpha / 2}^{K}$, for the alternative $H_{1}^{A K}$;

$\min _{k \leq K} T_{j}^{A}\left(x_{k}\right)<-M_{\infty, \alpha}^{K}$, for the alternative $H_{1 l}^{A K}$;

$\max _{k \leq K} T_{j}^{A}\left(x_{k}\right)>M_{\infty, \alpha}^{K}$, for the alternative $H_{1 r}^{A K} ;$ 
where $M_{\infty, \alpha}^{K}$ is the $1-\alpha$ percentile of $M_{\infty}^{K}$ tabulated by Stoline and Ury (1979):

Remark 3.1 The DD test compares distributions at a finite number of grid points. Various studies examine the choice of grid points. For example, Tse and Zhang (2004) show that an appropriate choice of $K$ for reasonably large samples ranges from 6 to 15 . Too few grids will miss information about the distributions between any two consecutive grids (Barrett and Donald, 2003), and too many grids will be said to violate the independence assumption required by the SMM distribution (Richmond, 1982). To make the comparisons comprehensive without too much violating the independence assumption, Fong, et al. (2005), Gasbarro, et al. (2007), and Wong, et al. (2008) suggest to use the 10 major partitions with 10 minor partitions within any two consecutive major partitions in each comparison and draw statistical inference based on the $S M M$ distribution for $K=10$ and infinite degrees of freedom.

As we have pointed out earlier that the limiting distributions of $T_{j}^{A}(x)$ are not independent even for two points partition. More importantly, the limiting correlations would depend on the null in which distributions $F=G$. To solve these problems, we suggest to investigate the limiting distribution of $T_{j}^{A}(x), a<x<b$, regarded as a stochastic process. To do so, we establish the following theorem.

Theorem 3.1 Let $\left\{f_{i}\right\}\left(i=1,2, \cdots, N_{f}\right)$ and $\left\{g_{i}\right\}\left(i=1,2, \cdots, N_{g}\right)$ be random observations drawn from the independent random variables $Y$ and $Z$, with continuous distribution functions $F$ and $G$, respectively. Under the null hypothesis $F \equiv G$, the $j^{\text {th }}$ order ASD test statistics, $T_{j}^{A}(x)(j=1$, 2 and 3), weakly tends to a limiting Gaussian process with mean 0 , variance 1 , and correlation function $r^{A}(x, y)$ in which for the case $j=1$, we get

$$
r_{1}^{A}(x, y)=\frac{F(x \wedge y)-F(x) F(y)}{\sqrt{F(x) F(y)(1-F(x))(1-F(y))}},
$$


and for the case $j>1$, we have

$$
r^{A}(x, y)=\frac{\int_{a}^{x} \int_{a}^{y}(x-t)^{j-2}(y-s)^{j-2}(F(t \wedge s)-F(t) F(s)) d t d s}{\sqrt{V_{j}^{A}(x) V_{j}^{A}(y)}},
$$

where $\hat{H}_{j}^{A}(x)$ and $\hat{V}_{j}^{A}(x)$ for $H=F$ and $G$ are defined in (3.2),

$$
V_{1}^{A}(x)=F(x)(1-F(x))
$$

and

$$
V_{j}^{A}(x)=\int_{a}^{x} \int_{a}^{x}(x-t)^{j-2}(x-s)^{j-2}(F(t \wedge s)-F(t) F(s)) d t d s
$$

Based on this theorem, to test the hypotheses in (3.1), we propose to reject the null hypothesis $H_{0}^{A}$ if

$$
\begin{gathered}
\max _{a<x<b}\left|T_{j}^{A}(x)\right|>M_{\alpha / 2}^{A}, \text { for the alternative } H_{1}^{A} \\
\min _{a<x<b} T_{j}^{A}(x)<-M_{\alpha}^{A}, \text { for the alternative } H_{1 l}^{A} \\
\max _{a<x<b} T_{j}^{A}(x)>M_{\alpha}^{A}, \text { for the alternative } H_{1 r}^{A}
\end{gathered}
$$

in which we suggest to obtain the critical values $M_{\alpha}^{A}$ by a bootstrap approach that will be described later in the paper.

In many cases, the asset random variables $Y$ and $Z$ are not independent of each other. Assume that they have a joint distribution $Q(y, z)$ with marginal distributions $F$ and $G$. Suppose that the sample is drawn in a way that $\left\{\left(f_{i}, g_{i}\right), i=1, \cdots, m, f_{k}, g_{l}, k=\right.$ $\left.m+1, \cdots, N_{f} ; l=m+1, \cdots, N_{g}\right\}$ are mutually independent. In such a case, the covariance of $\hat{F}_{j}^{A}(x)$ and $\hat{G}_{j}^{A}(x)$ can be estimated by

$$
\hat{V}_{F G_{j}}^{A}(x)=\frac{1}{N_{f} N_{g}((j-1) !)^{2}} \sum_{i=1}^{m}\left(x-f_{i}\right)_{+}^{j-1}\left(x-g_{i}\right)_{+}^{j-1}-\frac{m}{N_{f} N_{g}} \hat{F}_{j}^{A}(x) \hat{G}_{j}^{A}(x) .
$$

Hence, the variance of $\hat{F}_{j}^{A}(x)-\hat{G}_{j}^{A}(x)$ can be estimated by

$$
\hat{V}_{j}^{A}(x)=\hat{V}_{F_{j}}^{A}(x)+\hat{V}_{G_{j}}^{A}(x)-2 \hat{V}_{F G_{j}}^{A}(x),
$$


in which the integrals $F_{j}{ }^{A}$ and $G_{j}{ }^{A}$ are defined in (2.1) for $j=1,2$ and 3. Thereafter, one may define the test statistic $T_{j}^{A}(x)$ by (3.2) with the new $\hat{V}_{j}^{A}(x)$ defined in (3.4). We summarize the result in the following theorem:

Theorem 3.2 Let $\left\{f_{i}\right\}\left(i=1,2, \cdots, N_{f}\right)$ and $\left\{g_{i}\right\}\left(i=1,2, \cdots, N_{g}\right)$ be random observations drawn from the dependent random variables $Y$ and $Z$, with continuous joint distribution function $Q(x, y)$ and marginal distribution functions $F$ and $G$, respectively. Suppose the samples are drawn as described earlier; that is, we assume that $\left(f_{i}, g_{i}\right) ; i=$ $1, \cdots, m$ are pairwisely drawn and the rest are independent. Suppose that $m /\left(N_{f}+N_{g}\right) \rightarrow$ $\lambda$. Then, under the null hypothesis $F \equiv G$, the $j^{\text {th }}$ order ASD test statistic, $T_{j}^{A}(x)$ $(j=1,2$ and 3$)$, weakly tends to a limiting Gaussian process with mean 0, variance 1, and correlation function $r_{j \text {, dep }}^{A}(x, y)$ in which for the case $j=1$, we get

$$
r_{1, \text { dep }}^{A}(x, y)=\frac{F(x \wedge y)-\lambda Q(x, y)-(1-2 \lambda) F(x) F(y)}{\sqrt{F(x) F(y)(1-F(x))(1-F(y))}},
$$

and for the case $j>1$, we have

$$
r_{j, d e p}^{A}(x, y)=\frac{V_{j}^{A}(x, y)}{\sqrt{V_{j}^{A}(x) V_{j}^{A}(y)}}
$$

where $\hat{H}_{j}^{A}(x)$ and $\hat{V}_{j}^{A}(x)$ for $H=F$ and $G$ are defined in (3.3) and (3.4), respectively, and

$$
\begin{aligned}
V_{j}^{A}(x) & =\int_{a}^{x} \int_{a}^{x}(x-t)^{j-2}(x-s)^{j-2}(F(t \wedge s)-F(t) F(s)) d t d s \quad \text { and } \\
V_{j}^{A}(x, y) & =\int_{a}^{x} \int_{a}^{y}(x-t)^{j-2}(y-s)^{j-2}[(F(t \wedge s)-\lambda(Q(t, s)+Q(s, t))-(1-2 \lambda) F(t) F(s))] d t d s .
\end{aligned}
$$

\subsection{DSD Tests for Risk Seekers}

In this paper, we modify the DD test for risk averters to be DD test statistic for risk seekers and we call it the descending DD test statistic. Let $\left\{f_{i}\right\}\left(i=1,2, \cdots, N_{f}\right)$ and $\left\{g_{i}\right\}\left(i=1,2, \cdots, N_{g}\right)$ be observations drawn from the independent random variables $Y$ 
and $Z$ with distribution functions $F$ and $G$, respectively. The integrals $F_{j}^{D}$ and $G_{j}^{D}$ for $F$ and $G$ are defined in (2.1) for $j=1,2$ and 3. For a grid of pre-selected points $\left\{x_{k}\right.$, $k=1, \cdots, K\}$, the $j^{\text {th }}$ order descending DD test statistic, $T_{j}^{D}(x)(j=1,2$ and 3$)$, is:

$$
T_{j}^{D}(x)=\frac{\hat{F_{j}^{D}}(x)-\hat{G}_{j}^{D}(x)}{\sqrt{\hat{V}_{j}^{D}(x)}},
$$

where

$$
\begin{aligned}
\hat{V}_{j}^{D}(x) & =\hat{V}_{F_{j}}^{D}(x)+\hat{V}_{G_{j}}^{D}(x), \\
\hat{H}_{j}^{D}(x) & =\frac{1}{N_{h}(j-1) !} \sum_{i=1}^{N_{h}}\left(h_{i}-x\right)_{+}^{j-1}, \\
\hat{V}_{H_{j}}^{D}(x) & =\frac{1}{N_{h}}\left[\frac{1}{N_{h}((j-1) !)^{2}} \sum_{i=1}^{N_{h}}\left(h_{i}-x\right)_{+}^{2(j-1)}-\hat{H}_{j}^{D}(x)^{2}\right], H=F, G ; h=f, g ;
\end{aligned}
$$

in which the integrals $F_{j}{ }^{D}$ and $G_{j}{ }^{D}$ are defined in (2.1) for $j=1,2$ and 3 . For $k=$ $1, \cdots, K$, the following hypotheses are tested for risk seekers:

$$
\begin{aligned}
& H_{0}^{D}: F_{j}^{D}\left(x_{k}\right)=G_{j}^{D}\left(x_{k}\right) \text { for all } x_{k} ; \\
& H_{1}^{D}: F_{j}^{D}\left(x_{k}\right) \neq G_{j}^{D}\left(x_{k}\right) \text { for some } x_{k} ; \\
& H_{1 l}^{D}: F_{j}^{D}\left(x_{k}\right) \geq G_{j}^{D}\left(x_{k}\right) \text { for all } x_{k}, F_{j}^{D}\left(x_{k}\right)>G_{j}^{D}\left(x_{k}\right) \text { for some } x_{k} ; \\
& H_{2 r}^{D}: F_{j}^{D}\left(x_{k}\right) \leq G_{j}^{D}\left(x_{k}\right) \text { for all } x_{k} F_{j}^{D}\left(x_{k}\right)<G_{j}^{D}\left(x_{k}\right) \text { for some } x_{k} .
\end{aligned}
$$

Under the null hypothesis $H_{0}^{D}$, one may believe that $T_{j}^{D}$ is asymptotically distributed as the studentized maximum modulus (SMM) distribution (Richmond, 1982). Similar to the argument we correct the ASD test in last subsection, this is not correct and we correct the theory for the DSD test as follows:

To implement the DD test, $T_{j}^{D}$, for risk seekers for $j=1,2,3$, one should test the following hypothesis at each grid point being computed:

$$
H_{0}^{D}: F_{j}^{D} \equiv G_{j}^{D}
$$


against three alternatives

$$
\begin{array}{ll}
H_{1}^{D}: & F \not_{j}^{D} G, \text { two sided test, } \\
H_{1 l}^{D}: & F \succ_{j}^{D} G_{j}, \text { one sided test, } \\
H_{1 r}^{D}: & F \prec_{j}^{D} G, \quad \text { one sided test. }
\end{array}
$$

The three hypotheses are equivalent to

$$
\begin{array}{ll}
H_{1}^{D}: & F_{j}^{D}(x) \neq G_{j}^{D}(x), \text { for some } x, \\
H_{1 l}^{D}: & F_{j}^{D}(x) \geq G_{j}^{D}(x), \forall x \text { and the inequality is strict for at least one } x, \\
H_{1 r}^{D}: & F_{j}^{D}(x) \leq G_{j}^{D}(x), \forall x \text { and the inequality is strict for at least one } x .
\end{array}
$$

Using a similar technique as the development of Theorem 3.1, we obtain the following theorem for DSD test:

Theorem 3.3 Let $\left\{f_{i}\right\}\left(i=1,2, \cdots, N_{f}\right)$ and $\left\{g_{i}\right\}\left(i=1,2, \cdots, N_{g}\right)$ be random observations drawn from the independent random variables $Y$ and $Z$, with continuous distribution functions $F$ and $G$, respectively. Under the null hypothesis $F \equiv G$, the $j^{\text {th }}$ order DSD test statistic, $T_{j}^{D}(x)(j=1$, 2 and 3), weakly tends to a limiting Gaussian process with mean 0 , variance 1 , and correlation function $r^{A}(x, y)$ in which for the case $j=1$, we get

$$
r_{1}^{D}(x, y)=\frac{F(x \wedge y)-F(x) F(y)}{\sqrt{F(x) F(y)(1-F(x))(1-F(y))}},
$$

and for the case $j>1$, we have

$$
r^{D}(x, y)=\frac{\int_{x}^{b} \int_{y}^{b}(t-x)^{j-2}(s-y)^{j-2}(F(t \wedge s)-F(t) F(s)) d t d s}{\sqrt{V_{j}^{D}(x) V_{j}^{D}(y)}},
$$

where $\hat{H}_{j}^{D}(x)$ and $\hat{V}_{j}^{D}(x)$ are defined in (3.5) for $H=F$ and $G$,

$$
V_{1}^{D}(x)=F(x)(1-F(x))
$$


and

$$
V_{j}^{D}(x)=\int_{x}^{b} \int_{x}^{b}(t-x)^{j-2}(s-x)^{j-2}(F(t \wedge s)-F(t) F(s)) d t d s
$$

The proof of this theorem is given in Appendix. Based on this theorem, to test the hypotheses (3.6), we propose to reject the null hypothesis $H_{0}^{D}$ if

$$
\begin{aligned}
& \max _{a<x<b}\left|T_{j}^{D}(x)\right|>M_{\alpha / 2}^{D}, \text { for the alternative } H_{1}^{D} \\
& \max _{a<x<b} T_{j}^{D}(x)>M_{\alpha}^{D}, \text { for the alternative } H_{1 l}^{D} \\
& \min _{a<x<b} T_{j}^{D}(x)<-M_{\alpha}^{D}, \text { for the alternative } H_{1 r}^{D}
\end{aligned}
$$

where critical values $M_{\alpha}^{D}$ is suggested to be found by a bootstrap approach which will be described later in our paper.

Similar to the case of the ASD tests, the asset random variables $Y$ and $Z$ may not be independent of each other. Now, we assume that the samples have the same structure as described in last subsection. In such a case, the covariance of $\hat{F}_{j}^{D}(x)$ and $\hat{G}_{j}^{D}(x)$ can be estimated by

$$
\hat{V}_{F G_{j}}^{D}(x)=\frac{1}{N_{f} N_{g}((j-1) !)^{2}} \sum_{i=1}^{m}\left(f_{i}-x\right)_{+}^{j-1}\left(g_{i}-x\right)_{+}^{j-1}-\frac{m}{N_{f} N_{g}} \hat{F}_{j}^{D}(x) \hat{G}_{j}^{D}(x) .
$$

Hence, the variance of $\hat{F}_{j}^{D}(x)-\hat{G}_{j}^{D}(x)$ can be estimated by

$$
\hat{V}_{j}^{D}(x)=\hat{V}_{F_{j}}^{D}(x)+\hat{V}_{G_{j}}^{D}(x)-2 \hat{V}_{F G_{j}}^{D}(x) .
$$

Then, one may define the test statistic $T_{j}^{D}(x)$ by $(3.5)$ with the newly defined $\hat{V}_{j}^{D}(x)$. The proof of Theorem 3.3 is given in the Appendix.

Theorem 3.4 Let $\left\{f_{i}\right\}\left(i=1,2, \cdots, N_{f}\right)$ and $\left\{g_{i}\right\}\left(i=1,2, \cdots, N_{g}\right)$ be random observations drawn from the dependent random variables $Y$ and $Z$, with continuous joint distribution function $Q(x, y)$ and marginal distribution functions $F$ and $G$, respectively. Suppose the samples are drawn as described earlier; that $i s$, we assume that $\left(f_{i}, g_{i}\right) ; i=$ 
$1, \cdots, m$ are pairwisely drawn and the rest are independent. Suppose that $m /\left(N_{f}+N_{g}\right) \rightarrow$ $\lambda$. Then, under the null hypothesis $F \equiv G$, the $j^{\text {th }}$ order DSD test statistic, $T_{j}^{D}(x)$ $(j=1,2$ and 3$)$, weakly tends to a limiting Gaussian process with mean 0, variance 1, and correlation function $r_{j \text {, dep }}^{D}(x, y)$ in which for the case $j=1$, we get

$$
r_{1, \text { dep }}^{D}(x, y)=\frac{F(x \wedge y)-\lambda Q(x, y)-(1-2 \lambda) F(x) F(y)}{\sqrt{F(x) F(y)(1-F(x))(1-F(y))}},
$$

and for the case $j>1$, we have

$$
r_{j, \text { dep }}^{D}(x, y)=\frac{V_{j}^{D}(x, y)}{\sqrt{V_{j}^{D}(x) V_{j}^{D}(y)}},
$$

where $\hat{H}_{j}^{D}(x)$ and $\hat{V}_{j}^{D}(x)$ for $H=F$ and $G$ are defined in (3.3) and (3.7), respectively, and obtain

$$
\begin{aligned}
V_{j}^{D}(x) & =\int_{x}^{b} \int_{x}^{b}(t-x)^{j-2}(s-x)^{j-2}(F(t \wedge s)-F(t) F(s)) d t d s \\
V_{j}^{D}(x, y) & =\int_{x}^{b} \int_{y}^{b}(t-x)^{j-2}(s-y)^{j-2}[(F(t \wedge s)-\lambda(Q(t, s)+Q(s, t))-(1-2 \lambda) F(t) F(s))] d t d s .
\end{aligned}
$$

\section{Determination of Critical Values}

In this section, we discuss only the situation in which the variables are independent. The situation in which the variables are dependent could be obtained similarly. Suppose the variables being examined are independent and the sample series $\left\{f_{i}, i=1,2, \cdots, N_{f}\right\}$ and $\left\{g_{i}, i=1,2, \cdots, N_{g}\right\}$ are iid. We draw two resamples $\left\{f_{i}^{*}, i=1,2, \cdots, N_{f}\right\}$ and $\left\{g_{i}^{*}, i=1,2, \cdots, N_{g}\right\}$ from the pooled sample $\left\{f_{(i)}, g_{(j)}, i=1,2, \cdots, N_{f}, j=1,2, \cdots, N_{g}\right\}$,

the $D D$ test statistics $\left(\hat{T}_{j}^{A}\right.$ and $\left.\hat{T}_{j}^{D}\right)(j=1,2,3)$ defined in $(3.2)$ and (3.5) can then be bootstrapped. Using this method, one can approximate the null distribution of the test statistics. The details to obtain the critical values of statistic $\hat{T}_{j}^{A}$ and $\hat{T}_{j}^{D}, j=1,2,3$, are as follows: 
Step 1 Draw a sample set $\left\{f_{i}^{*}, i=1,2, \cdots, N_{f}\right\}$ from $\left\{f_{i}, g_{j}, i=1,2, \cdots, N_{f}, j=1,2, \cdots, N_{g}\right\}$ with replacement and draw another sample set $\left\{g_{i}^{*}, i=1,2, \cdots, N_{g}\right\}$ in the same way.

Step 2 Compute: $A_{j}=\hat{T}_{j}^{* A}(x)=\left[\hat{F_{j}^{A}}(x)-\hat{G}_{j}^{A}(x)\right] / \sqrt{\hat{V}_{j}^{A}(x)}$, and $D_{j}=\hat{T}_{j}^{* D}(x)=$ $\left[\hat{F}_{j}^{D}(x)-\hat{G}_{j}^{D}(x)\right] / \sqrt{\hat{V}_{j}^{D}(x)}$, where $\hat{H}_{j}(x)$ and $\hat{V}_{j}(x)$ for $H=F$ and $G$, respectively, are defined in (3.2). Repeat this process $N$ times.

Step 3 Find $A_{j}(\alpha)$ such that $\sharp\left\{\left|A_{j k}\right| \geq A_{j}(\alpha), k \leq N\right\}=\left[N_{\alpha}\right]$; that is, the $\alpha$ percentile of the distribution of $\hat{T}_{j}^{* A}$.

Step 4 Find $D_{j}(\alpha)$ such that $\sharp\left\{\left|D_{j k}\right| \geq D_{j}(\alpha), k \leq N\right\}=\left[N_{\alpha}\right]$; that is, the $\alpha$ percentile of the distribution of $\hat{T}_{j}^{* D}$.

We make the following remarks in obtaining the critical values:

Remark 4.1 The samples $\left\{f_{i}^{*}, g_{j}^{*}, i=1,2, \cdots, N_{f}, j=1,2, \cdots, N_{g}\right\}$ are iid random samples with distribution function $F_{N_{f}+N_{g}}^{*}=\frac{N_{f}}{N_{f}+N_{g}} F_{N_{f}}+\frac{N_{g}}{N_{f}+N_{g}} G_{N_{g}}$. So, under the null hypothesis $F^{*}=F=G$, the sample sets $\left\{f_{i}^{*}, g_{j}^{*}, i=1,2, \cdots, N_{f}, j=1,2, \cdots, N_{g}\right\}$ and $\left\{f_{i}, g_{j}, i=1,2, \cdots, N_{f}, j=1,2, \cdots, N_{g}\right\}$ are almost the same as the samples which come from the same distribution only if the sum of $N_{f}$ and $N_{g}$ is large enough. Under this situation, the critical value obtained by using the above procedure will be almost the same as the real critical point value. But under the relative hypothesis, because the sample sets $\left\{f_{i}, i=1,2, \cdots, N_{f}\right\}$ and $\left\{g_{j}, j=1,2, \cdots, N_{g}\right\}$ come from different distributions $F$ and $G$, respectively, the absolute value of $\hat{T}_{j}$ with the sample set $\left\{f_{i}^{*}, g_{j}^{*}, i=1,2, \cdots, N_{f}, j=\right.$ $\left.1,2, \cdots, N_{g}\right\}$ could be different from the real critical point value.

Remark 4.2 Start with $N=10000, N_{f}=200, N_{g}=200$, and $\alpha=0.05$. Given that $F$ and $G$ are from some specific distributions, we compare the probability of the error of the first kind and the power for some varieties for pairs of $F$ and $G$. For example, suppose 
$F=N(0,1), G=N(0,1), N(0.1,1), N(0.2,1), N(0.3,1), N(0.4,1)$, and $N(0.5,1)$, respectively, we obtain the simulation results in Table 1. From the table, one could easily conclude that when $F$ and $G$ are further apart, the difference between the errors of the first kind and the power become greater.

Table 1: the error of the first kind and the power of SD tests

\begin{tabular}{|c|c|c|c|c|c|c|}
\hline \multicolumn{7}{|c|}{ ASD test } \\
\hline $\mathrm{j}=1$ & $\mathrm{~N}(0,1)$ & $\mathrm{N}(0.1,1)$ & $\mathrm{N}(0.2,1)$ & $\mathrm{N}(0.3,1)$ & $\mathrm{N}(0.4,1)$ & $\mathrm{N}(0.5,1)$ \\
$\mathrm{N}(0,1)$ & 0.057 & 0.139 & 0.405 & 0.700 & 0.934 & 0.993 \\
$\mathrm{j}=2$ & $\mathrm{~N}(0,1)$ & $\mathrm{N}(0.1,1)$ & $\mathrm{N}(0.2,1)$ & $\mathrm{N}(0.3,1)$ & $\mathrm{N}(0.4,1)$ & $\mathrm{N}(0.5,1)$ \\
$\mathrm{N}(0,1)$ & 0.060 & 0.122 & 0.430 & 0.733 & 0.956 & 0.997 \\
$\mathrm{j}=3$ & $\mathrm{~N}(0,1)$ & $\mathrm{N}(0.1,1)$ & $\mathrm{N}(0.2,1)$ & $\mathrm{N}(0.3,1)$ & $\mathrm{N}(0.4,1)$ & $\mathrm{N}(0.5,1)$ \\
$\mathrm{N}(0,1)$ & 0.036 & 0.074 & 0.085 & 0.174 & 0.208 & 0.423 \\
\hline \multicolumn{7}{|c|}{$\mathrm{DSD}$ test } \\
\hline $\mathrm{j}=1$ & $\mathrm{~N}(0,1)$ & $\mathrm{N}(0.1,1)$ & $\mathrm{N}(0.2,1)$ & $\mathrm{N}(0.3,1)$ & $\mathrm{N}(0.4,1)$ & $\mathrm{N}(0.5,1)$ \\
$\mathrm{N}(0,1)$ & 0.0499 & 0.1227 & 0.3566 & 0.6906 & 0.9156 & 0.9921 \\
$\mathrm{j}=2$ & $\mathrm{~N}(0,1)$ & $\mathrm{N}(0.1,1)$ & $\mathrm{N}(0.2,1)$ & $\mathrm{N}(0.3,1)$ & $\mathrm{N}(0.4,1)$ & $\mathrm{N}(0.5,1)$ \\
$\mathrm{N}(0,1)$ & 0.0542 & 0.1434 & 0.4191 & 0.7726 & 0.9577 & 0.9965 \\
$\mathrm{j}=3$ & $\mathrm{~N}(0,1)$ & $\mathrm{N}(0.1,1)$ & $\mathrm{N}(0.2,1)$ & $\mathrm{N}(0.3,1)$ & $\mathrm{N}(0.4,1)$ & $\mathrm{N}(0.5,1)$ \\
$\mathrm{N}(0,1)$ & 0.0511 & 0.1362 & 0.4097 & 0.7594 & 0.9495 & 0.9962 \\
\hline
\end{tabular}

Note: ASD test is defined in (3.2) while DSD test is defined in (3.5). 


\section{Empirical Results}

In this section, we will illustrate the applicability of the SD statistics for both risk averters and risk seekers developed in this paper to analyze the dominance relationship between the Chinese and US stock markets in the entire period as well as the sub-periods before and after the financial crises, including the internet bubble and the recent sub-prime crisis. We are also interested in studying their preferences in these markets in the bull runs as well as in the bear markets. To do so, we apply the modified DD test statistics developed above to examine the daily returns of both SSE and S\&P 500 for the full sample period (02/01/1996 to 19/05/2011) and the 5 sub-periods: $01 / 1996$ to $03 / 2000$, $03 / 2000$ to $10 / 2002,10 / 2002$ to $10 / 2007,10 / 2007$ to $11 / 2008$, and $11 / 2008$ to $05 / 2011$. SSE denotes the Shanghai Stock Exchange Composite Index, while S\&P 500 denotes the Standard \& Poor 500 index, a free-float capitalization-weighted index of the prices of 500 large-cap common stocks actively traded in the USA.

The Chinese stock markets, initiated in early 1990's, have been expanding tremendously in the past decade. China has experienced dramatic economic growth in the past decade. Its average annual growth rate is about $9 \%$, much higher than that of the world economy. As one important component of the Chinese economy, Chinese stock markets, have also expanded rapidly. There are many studies that have analyzed the Chinese and USA stock markets. For example, Chakravarty et al. (1998) report the bivariate return correlations among the Chinese stock indices, as well as the U.S. market indices, and suggest that the Chinese market is still isolated.

We now ready to discuss our analysis for the performance of the daily returns of Chinese (SSE) and USA (S\&P) stock markets for risk averters as well as for risk seekers in the full sample period as well as all the sub-periods. To analyze the preferences for risk averters, we adopt the modified DD statistic $T_{j}^{A}$ (we call it ASD test) for risk averters for $j=1 ; 2$, and 3 where $T_{j}^{A}$ is defined in (3.2) for $j=1,2$, and 3 with $F$ and $G$ be the 
CDFs of the returns of $S \& P$ and $S S E$, respectively. We display the results in Table 2. In addition, we plot the ASD tests together with the CDFs, $F$ and $G$, of the daily returns of $S \& P$ and $S S E$ in Figure 2. On the other hand, to analyze the preferences for risk seekers, we employ the modified DD statistic $T_{j}^{D}$ (we call it DSD test) for risk seekers for $j=1,2$, and 3 where $T_{j}^{D}$ is defined in (3.5) for $j=1 ; 2$, and 3 with $F=S \& P$ and $G=S S E$. We exhibit the results in Table 3. In addition, we plot the DSD tests together with the DSD integrals $F_{1}^{D}$ and $G_{1}^{D}$ defined in (2.1) in Figure 3. At last, we summarize all SD relationship between SSE and S\&P 500 for the full sample period as well as all the 5 sub-periods in Table 4.

\subsection{First Order Stochastic Dominance Analysis}

We first our analysis for the performance of the daily returns of the Chinese and USA stock markets for risk averters in the full sample period. To minimize the Type II error and to avoid SD (Leshno and Levy, 2002), we use a 5 per cent cut-off point ${ }^{4}$ to the proportion of the values of the test statistic in (3.2) for SSE and S\&P to draw statistical inferences. Using the 5 per cent cut-off point, if S\&P dominates SSE, one should find at least 5 per cent of $T_{j}^{A}$ to be significantly negative and no portion of $T_{j}^{A}$ to be significantly positive. The reverse holds if SSE dominates S\&P. From Table 2, we find that 34.92 per cent (27.92 per cent) of $T_{1}^{A}$ is significantly positive (negative) for the entire period. Thus, the results lead us reject the hypothesis that S\&P stochastically dominates SSE or vice versa at the first order in the sense of ASD.

Together with the plot of the modified ASD test for risk averters exhibited in Figure 2 , the results from Table 2 show that $T_{1}^{A}$ is significantly negative in the downside risk and significantly positive in the upside profit, inferring that investors with increasing utility functions will prefer to invest in $\mathrm{S} \& \mathrm{P}$ when facing with the downside risk, whereas they

\footnotetext{
${ }^{4}$ We note that Leshno and Levy (2002) use an example of 1 per cent to state the problem of almost SD. We choose a more conservative 5 per cent cut-off point to avoid the problem of almost SD. The conclusion drawn in our paper holds if one uses any less conservative cut-off point, say 1 per cent.
} 
will prefer to invest in SSE in anticipation of the upside profit. This could also infer that investors prefer to invest in USA stock market in the bear markets and prefer to invest in Chinese stock market in the bull runs. Table 3 shows that 27.92 per cent (34.92 per cent) of $T_{1}^{D}$ is significantly positive (negative), leading us reject the hypothesis that S\&P stochastically dominates SSE or vice versa in the sense of FDSD. Together with the plot of the modified DSD test for risk seekers displayed in Figure 3, the results reveal that $T_{1}^{D}$ is significantly positive at the downside risk and significantly negative at the upside profit. This result draws the same inference as the result of $T_{1}^{A}$ that $\mathrm{S} \& \mathrm{P}$ is preferred to SSE on the downside risk and SSE is preferred on the upside profits. These results do not reject the market efficiency hypothesis. In order to explore whether the market is efficient and investors are rational, we need to examine the higher-order SD.

\subsection{Higher-Order Stochastic Dominance}

We now turn to examine whether there is any higher-order stochastic dominance relationship between the Chinese and USA stock markets. From Table 2, we observe that 89.57 per cent of the second-order modified ASD statistic $T_{2}^{A}$ is significantly negative and none of it is significantly positive at the 5 per cent bootstrap simulated critical level. Similarly, from Table 2, we find that 98.98 per cent of the third-order modified ASD statistic $T_{3}^{A}$ is significantly negative and none of it is significantly positive at the 5 per cent bootstrap simulated critical level. Hence, we conclude that there is a dominance of S\&P over SSE in terms of both SASD and TASD at the 5 per cent significant level, inferring that second- and third-order risk averters prefer to invest in the USA stock market rather than the Chinese stock market. In addition, we also apply the testing procedure by using $\max _{x}\left|T_{j}^{A}(x)\right|$. The inference drawn from this approach leads to the same conclusion.

From the results discussed in the above, one may follow the suggestion from Falk and Levy (1989) and others and conclude that the markets are not efficient and investors are 
not rational. In order to check whether the market is efficient and investors are rational, in this paper we suggest studying the preference of risk seekers.

\subsection{Preference of Risk Seekers}

In order to study the preferences of risk seekers between the Chinese and USA stock markets, we adopt the DSD theory and employ the DSD statistic, $T_{j}^{D}$, for risk seekers as stated in (3.5) for $j=2$ and 3 to conduct the analysis. From Table 3, we find that 96.42 per cent of $T_{2}^{D}$ is significantly negative and no portion of $T_{2}^{D}$ is significantly positive at the 5 per cent significant level for the whole sample period. This implies that SSE stochastically dominates S\&P in the sense of SDSD, and thus we conclude that secondorder risk seekers prefer SSE to S\&P for the whole sample period. Similarly, from Table

3, we find that 98.96 per cent of $T_{3}^{D}$ is significantly negative and no portion of $T_{3}^{D}$ is significantly positive at the 5 per cent level for the whole sample period. This implies that SSE stochastically dominates S\&P in the sense of TDSD, and thus third-order risk seekers prefer SSE to S\&P for the whole sample period.

Different from the conclusion drawn in the ASD test in which one could conclude that risk averters prefer to invest in the USA stock market rather than the Chinese stock market, our DSD analysis reveals the reverse preference for risk seekers that they are attracted to the Chinese stock market to maximize their expected utilities. Is there any inference on market efficiency and rationality from our findings? We will discuss the issue in next section.

\subsection{Robustness Analysis in the Sub-Periods}

We turn to investigate the preferences for risk averters and risk seekers in the Chinese and USA stock markets in each of the sub-periods. We first discuss their relationship in the sense of the first-order stochastic dominance.

Table 2 indicates that 36.9 per cent, 38.8 per cent, 30.3 per cent, 0 per cent, and 15.9 
per cent of $T_{1}^{A}$ are significantly positive in the first, second, third, fourth, and fifth subperiods, respectively, and all are occurred in the positive domain. On the other hand, 31.6 per cent, 4.0 per cent, 23.8 per cent, 25.3 per cent, and 0 per cent of $T_{1}^{A}$ are significantly negative for the first, second, third, fourth, and fifth sub-periods, respectively, and all are occurred in the negative domain. Since the analysis of the FDSD is the same as that of the FASD, we skip the discussion of the FDSD analysis. From our finding, we notice that only the first and the third sub-periods (both are in the bull runs) are similar to the ASD results for the entire period that the results lead us to reject the hypothesis that S\&P stochastically dominates SSE or vice versa in the sense of FSD. Thus, for the first and third sub-periods, the conclusion drawn from our FSD analysis is the same as that drawn for the entire period that there is no FSD between S\&P and SSE but S\&P is preferred to SSE on the downside risk and SSE is preferred on the upside profits.

For the second sub-period from March 9, 2000 to October 7, 2002, the period after the burst of internet bubble, investors may expect that the stock market in the USA does not perform well and the Chinese stock market will be doing better than the USA stock market. Our FSD analysis supports this argument. From Table 2, we find that 38.8 per cent of $T_{1}^{A}$ are significantly positive in the positive domain whereas 4.0 per cent of $T_{1}^{A}$ are significantly negative in the negative domain. One may infer that, same as those obtained in the first and third sub-periods, there is no FSD between S\&P and SSE but $\mathrm{S} \& \mathrm{P}$ is preferred to SSE on the downside risk and SSE is preferred on the upside profits. However, if one uses a 5 per cent cut-off point to exclude the possibility of almost SD (Leshno and Levy, 2002), one could claim that SSE stochastically dominates S\&P in the sense of FSD.

From FSD analysis in the period after the burst of the sub-prime crisis from October 16, 2007 to November 4, 2008, one may recommend investors to invest in the Chinese stock market but not to invest in the USA stock market when the USA is in a financial 
crisis. However, this may not be true. We turn to conduct our FSD analysis to these market during the period after the burst of the sub-prime crisis support this argument. From Table 2, we notice that no $T_{1}^{A}$ is significantly positive whereas it possesses 25.3 per cent to be significantly negative, inferring that, surprisingly, the S\&P stochastically dominates SSE in the sense of FSD and thus, investors should invest in the USA stock market rather than the Chinese stock market during the sub-prime crisis.

Furthermore, one may believe to invest in the USA stock market is better than the Chinese stock market since the investment is better even during the sub-prime crisis. Our $\mathrm{SD}$ analysis finds that this may not be true either. We make such inference from our SD analysis in the period during recovery after the burst of sub-prime crisis from November 4 , 2008 to May 19, 2011. Table 2 exhibits that 15.9 per cent of $T_{1}^{A}$ are significantly positive whereas none of it is significantly negative. One could conclude that SSE stochastically dominates the S\&P in the sense of FSD and thus, investors should invest in the Chinese stock market instead of investing in the USA stock market in the bull run during the recovery of the sub-prime crisis.

However, the situation is not that simple because though we find that SSE stochastically dominates the S\&P in the sense of FASD, our findings indicate that SSE does not stochastically dominate the S\&P in the sense of both SASD and TASD. One may believe that this result contradicts the hierarchical property for ASD that FASD implies SASD which, in turn, implies TASD and thus it is recommended that only the lowest dominance order of ASD is reported. However, our findings show that FASD does not imply SASD or TASD! We note that there is no contradiction. The hierarchical property does, in fact, hold for both ASD and DSD mathematically but this does not imply that the hierarchical property must hold statistically. Our finding in the fifth subperiod shows that it is possible that FASD does not imply SASD or TASD and thus in this paper we suggest that academics and practitioners report FSD, SSD, and TSD, in case, say, X stochastically 
dominates Y FASD, but not SASD and TASD. We suggest to call it X stochastically dominates Y marginally in the sense of FASD.

\section{Inference from our Findings}

Without identifying any risk index or any specific model, the SD rules can be used to determine whether there is any opportunity for arbitrage and whether the markets are efficient and investors are rational. We begin by examining arbitrage opportunities and then turn to the questions of market efficiency and investor rationality.

\subsection{Inference on Arbitrage Opportunity}

Jarrow (1986) and Falk and Levy (1989) claim that if FSD (FASD or FDSD) exists, under certain conditions, arbitrage opportunities also exist, and investors will increase their wealth and expected utilities if they shift from holding the dominated asset to the dominant one. However, Wong, et al. (2008) have shown that if FSD exists statistically, arbitrage opportunities may not exist, but investors can increase their expected wealth as well as their expected utilities if they shift from holding the dominated asset to the dominant one.

To check whether there is any arbitrage opportunity, one can apply the FSD analysis to the assets for comparison. In our analysis discussed in Section 5.1, although we find that S\&P FSD dominates SSE in the downside returns while SSE FSD dominates S\&P in the upside returns, S\&P does not FSD dominate SSE over the entire distribution and vice versa. This implies that there is no arbitrage opportunity in the USA and Chinese stock markets over the entire period studied in this paper.

However, in Section 5.5, we find that (1) in the second sub-period from March 9, 2000 to October 7, 2002, the period after the burst of internet bubble, if one adopts a 5 per cent cut-off point to exclude the possibility of almost SD, one could claim that SSE 
stochastically dominates S\&P in the sense of FSD; (2) in the period after the burst of the sub-prime crisis from October 16, 2007 to November 4, 2008, the S\&P stochastically dominates SSE in the sense of FSD; and (3) in the period during recovery from the sub-prime crisis from November 4, 2008 to May 19, 2011, one could conclude that SSE stochastically dominates the S\&P marginally in the sense of FSD.

Could these findings infer that there are arbitrage opportunities in the USA and Chinese stock markets and these markets are not efficient? We will say, yes, there could exist some arbitrage opportunities in the USA and Chinese stock markets in some short periods and we will discuss the issue of market efficiency in the next subsection.

\subsection{Inference on Market Efficiency and Rationality}

In Section 5.5, in some short periods SSE stochastically dominates $\mathrm{S} \& \mathrm{P}$ in the sense of FSD, whereas in some other short periods, the first order dominance relationship reverses. Indeed, these results do infer that there are some arbitrage opportunities in some short periods of time. However, since there is no FSD for a long period in these markets, we will say that our FSD findings do not reject that markets are efficient and investors are rational.

However, in order to further explore whether the market is efficient and investors are rational, we need to examine the higher order of SD as studied in Section 5.2. If no SASD is found in the market containing $\mathrm{X}$ and $\mathrm{Y}$, this suggests that risk-averse investors are indifferent between $\mathrm{X}$ and $\mathrm{Y}$, so they will not switch $\mathrm{X}$ to $\mathrm{Y}$, or vice-versa, to increase their expected utility. In this situation, we claim that the market is rational and efficient. Similarly, if no TASD is found in the market containing $\mathrm{X}$ and $\mathrm{Y}$, this implies that riskaverse investors with DARA are indifferent between $\mathrm{X}$ and $\mathrm{Y}$. In this situation, we claim that the market is both rational and efficient.

Nonetheless, Falk and Levy (1989) claim that, given two assets, X and Y, if by switch- 
ing from $\mathrm{X}$ to $\mathrm{Y}$ (or by selling $\mathrm{X}$ short and holding $\mathrm{Y}$ long), an investor can increase expected utility, the market is inefficient. SSD does not imply any arbitrage opportunities, but it does imply the preference of one asset over another by risk-averse investors. For example, as we found in Section 5.2, S\&P stochastically dominates SSE in the sense of both SASD and TASD. Thus, one may not make an expected profit by switching from SSE to S\&P, but switching would allow risk-averse investors to increase their expected utility. In this situation, should we claim that the USA and Chinese stock markets are inefficient and investors are irrational?

This claim could be made if one believes that the markets only contain risk-averse investors. However, it is well known that the market could have other types of investors (see, for example, Friedman and Savage, 1948, Markowitz, 1952, Thaler and Johnson, 1990, Broll et al., 2010, and Egozcue et al., 2011 for more discussion). Under the assumption that the markets could contain more than one type of investors, such as risk averters as well as risk seekers, one could find that one asset dominates another asset by ASD but is dominated by that asset by DSD. These are exactly the findings we obtained in this paper: S\&P stochastically dominates SSE in the sense of both SASD and TASD, while SSE stochastically dominate S\&P strictly in the sense of SDSD and TDSD.

Thus, risk averters could prefer to invest in the USA stock market rather than the Chinese stock market, while risk seekers prefer to invest in the Chinese stock market rather than the USA market. Then, in equilibrium, the number of trades that risk averters, who go long in the USA market and/or short sell the Chinese stock market, would match the number of trades that risk seekers, who go long in the Chinese stock market and/or short sell the USA market. In this situation, there is no pressure to push up or down the indices in the USA and Chinese stock markets when both risk averters and risk seekers can attain what they seek. Under these conditions, we argue that the market remains efficient and investors are rational. 
At last, we note that, conceptually, market rationality within the SD framework is not different from the conventional concepts captured by some of the rational asset pricing models, such as the CAPM. The only difference is that the latter approach defines an abnormal return as an excess return adjusted to some specific risk measure, while SD market rationality tests employ the whole distribution of returns. Given the imprecise knowledge of the best model, the SD approach with fewer restrictions on both investors' preferences and return distributions seems to help us understand the markets better.

\section{Concluding Remarks}

There are two basic approaches to the problem of portfolio selection under uncertainty. One approach is based on the concept of utility theory (see, for example, Markowitz (1952a), Post and Levy (2005), and the references therein for more information). Several SD test statistics ${ }^{5}$ have been developed by using this approach. This approach offers a mathematically rigorous treatment for portfolio selection. The other is the mean-risk (MR) analysis. ${ }^{6}$ In this approach, the portfolio choice is made with respect to two measures: the expected portfolio mean return and portfolio risk. A portfolio is preferred if it has higher expected return and smaller risk. There are convenient computational recipes and geometric interpretations of the trade-off between the two measures. A disadvantage of the latter approach is that it is derived by assuming the von Neumann-Morgenstern quadratic utility function and that returns are normally distributed (Feldstein, 1969). Thus, it cannot capture the richness of the former. In addition, the SD test statistics are superior to the MR test statistics because the conclusions drawn by these SD test statistics between the assets being examined could be used by investors to compare their expected utility on these assets since they do not require investors to possess a quadratic

\footnotetext{
${ }^{5}$ see, e.g. Schechtman, et al. (2008) and the references therein for more information.

${ }^{6}$ See, for example, Leung and Wong (2008) and Bai, el al. $(2009,2012)$ and the references therein for more discussion.
} 
utility function nor any form of the distribution for the assets being analyzed.

We note that Post and Versijp (2007) have developed a new SD test for multiple comparisons. Recently, Egozcue and Wong (2010) present a general theory and a unifying framework for determining the second-order SD efficient set. Further research could include extending the DD tests to determine the SD efficient set statistically. In addition, Lam, et al. (2010, 2012) develop a pseudo-Bayesian model to explain some anomalies such as the short-run underreaction and long-run overreaction. Further research could also apply the DD tests to explore the short-run underreaction and long-run overreaction phenomena. In addition, the theory of SD has been widely used to explain well-known economic and financial phenomena or financial anomalies and used to test for market efficiency (Lean, et al., 2010; Chan, et al., 2012). It could also be used to assist investors for decision making. 


\section{Appendix}

\section{Proof of Theorem 3.3:}

Without loss of generality, we assume $N_{f}=N_{g}=N$ in the proof. According to empirical process theorem ${ }^{7}$, for any continuous distribution function $F(x)$, we have

$$
\sqrt{N}\left(F_{N}(x)-F(x)\right) \longrightarrow B(F(x))
$$

where $F_{N}$ is the empirical distribution of $N$ iid observations drawn from $F$ and $B(\cdot)$ is the standardized Brownian bridge on the interval $[0,1]$.

Recall that

$$
\hat{F}_{j}^{D}(x)=\frac{1}{N(j-1) !} \sum_{i=1}^{N}\left(f_{i}-x\right)_{+}^{j-1}=\frac{1}{(j-1) !} \int_{x}^{b}(t-x)^{j-1} d F_{N}(t) \text { if } a \leq x \leq b .
$$

Applying the empirical process theorem, for $j \geq 1$, we have

$$
\sqrt{N}\left(\hat{F}_{j}^{D}(x)-F_{j}^{D}(x)\right) \rightarrow \frac{1}{(j-1) !} \int_{x}^{b}(t-x)^{j-1} d B(F(t)) \text { if } a \leq x \leq b .
$$

Because $F_{j}^{D}(x)=G_{j}^{D}(x)$ under null hypothesis, and $\hat{F}_{j}^{D}(x)$ and $\hat{G}_{j}^{D}(x)$ are independent, we get

$$
\sqrt{N}\left(\hat{F}_{j}^{D}(x)-\hat{G}_{j}^{D}(x)\right) \rightarrow \frac{\sqrt{2}}{(j-1) !} \int_{x}^{b}(t-x)^{j-1} d B(F(t)) \text { if } a \leq x \leq b .
$$

By the law of large numbers, with probability 1, one could easily find that

$$
\left\{\begin{array}{l}
N \hat{V}_{F G_{j}}^{D} \rightarrow 0 \\
N \hat{V}_{F_{j}}^{D} \rightarrow \frac{1}{((j-1) !)^{2}} \int_{a}^{x}(t-x)^{2 j-2} d F(t)-F_{j}^{D}(x)^{2}, \\
N \hat{V}_{G_{j}}^{D} \rightarrow \frac{1}{((j-1) !)^{2}} \int_{a}^{x}(t-x)^{2 j-2} d G(t)-G_{j}^{D}(x)^{2} .
\end{array}\right.
$$

Thereafter, under the null hypothesis, we get

$$
\begin{aligned}
N\left(\hat{V}_{F_{j}}^{D}+\hat{V}_{G_{j}}^{D}\right) & \rightarrow \frac{2}{((j-1) !)^{2}} \int_{x}^{b}(t-x)^{2 j-2} d F(t)-F_{j}^{D}(x)^{2} \\
& \stackrel{j>1}{=} \frac{2}{((j-2) !)^{2}} \int_{x}^{b} \int_{x}^{b}(t-x)^{j-2}(s-x)^{j-2}(F(t \wedge s)-F(t) F(s)) d t d s .
\end{aligned}
$$

\footnotetext{
${ }^{7}$ Readers may refer to Donsker (1952) and Wolfowitz (1954) for more information about empirical process theorem.
} 
Using integration by parts, when $j>1$, we have

$$
\frac{\sqrt{2}}{(j-1) !} \int_{x}^{b}(t-x)^{j-1} d B(F(t))=-\frac{\sqrt{2}}{(j-2) !} \int_{x}^{b}(t-x)^{j-2} B(F(t)) d t .
$$

Hence, when $j>1$, we obtain

$$
T_{j}^{D}(x) \rightarrow-\frac{\int_{x}^{b}(t-x)^{j-2} B(F(t)) d t}{\sqrt{\int_{x}^{b} \int_{x}^{b}(t-x)^{j-2}(s-x)^{j-2}(F(t \wedge s)-F(t) F(s)) d t d s}} \text { if } a \leq x \leq b .
$$

From this expression, we know that the limiting process of $T_{j}$ is Gaussian with mean 0 , variance 1 , and covariance function

$$
r_{j}^{D}(x, y)=\frac{\int_{x}^{b} \int_{a}^{y}(t-x)^{j-2}(s-y)^{j-2}(F(t \wedge s)-F(t) F(s)) d t d s}{\sqrt{\mathbb{V}_{j}^{D}(x) \mathbb{V}_{j}^{D}(y)}} \text { if } a \leq x, y \leq b,
$$

where $\mathbb{V}_{j}^{D}(x)=\int_{a}^{x} \int_{a}^{x}(t-x)^{j-2}(s-x)^{j-2}(F(t \wedge s)-F(t) F(s)) d t d s$. For the case $j=1$, we have $T_{1}^{D}(x, y)=\frac{B(F(x))}{\sqrt{F(x)-F^{2}(x)}}$. The limiting process is also Gaussian with mean 0 , variance 1 , and covariance function $r_{1}^{D}(x, y)=\frac{F(x \wedge y)-F(x) F(y)}{\sqrt{F(x) F(y)(1-F(x))(1-F(y))}}$ if $a<x, y<b$. 
Figure 1: Time series plot of SSE and S\&P500 from 02/01/1996 to 19/05/2011.

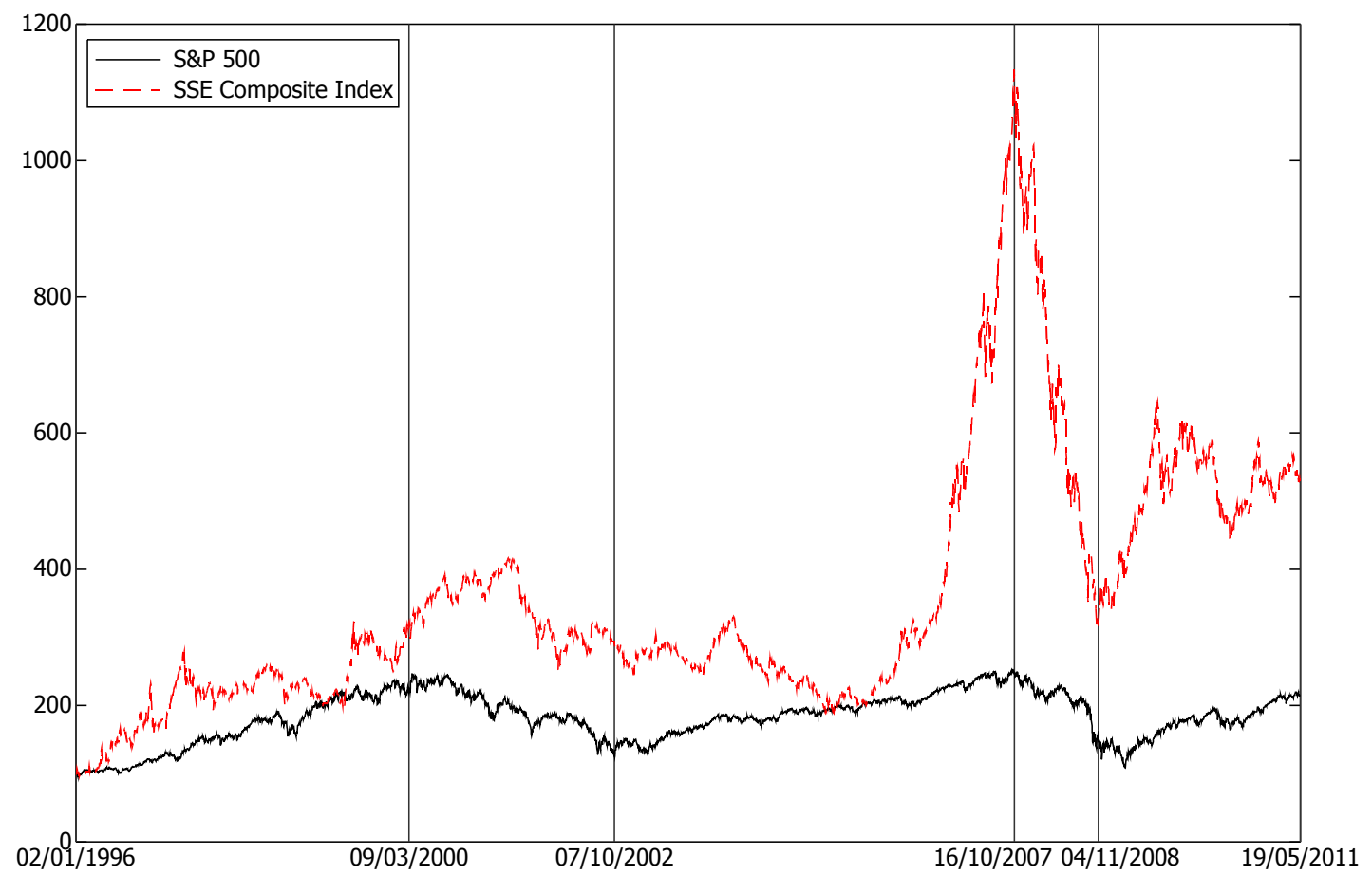

Figure 2: The modified ASD test statistics and the distribution functions $F$ and $G$ from 02/01/1996 to $19 / 05 / 2011$

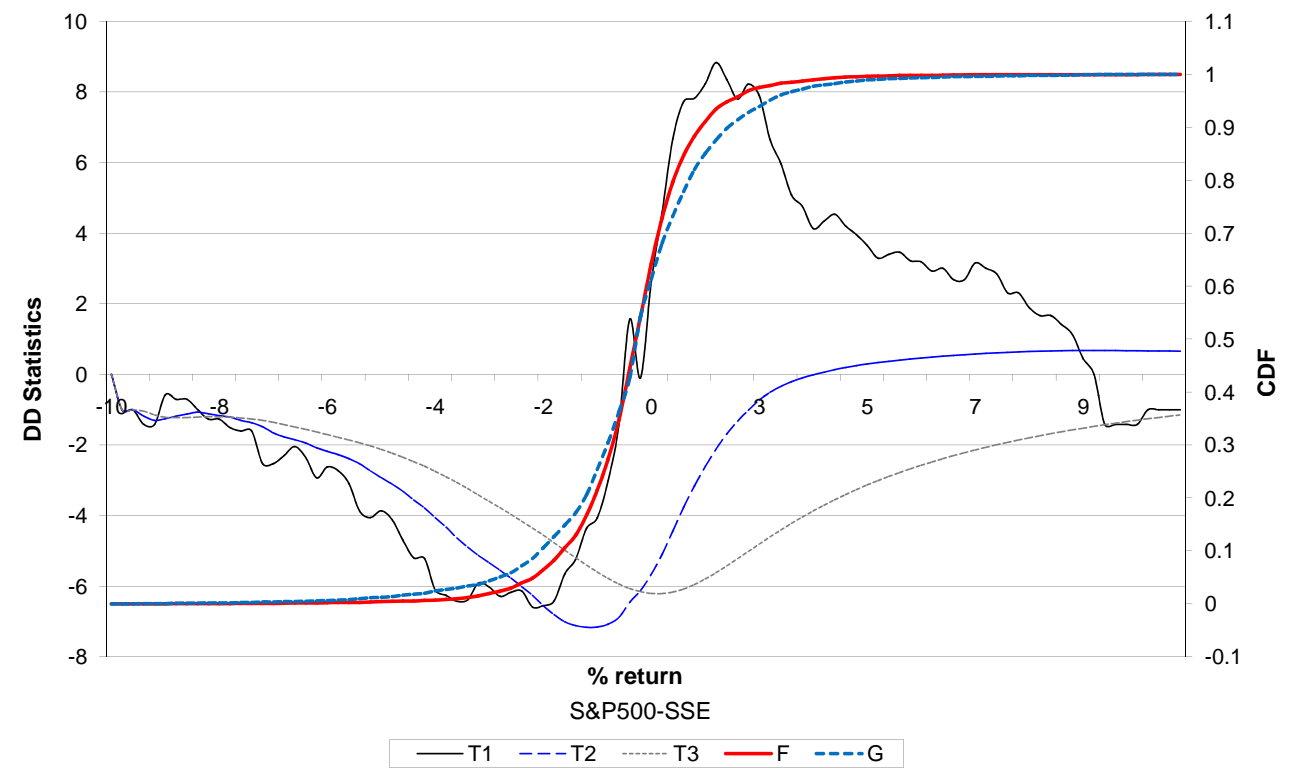

Note: $T j$ is the test statistic $T_{j}^{A}(x)$ defined in (3.2) for $j=1,2$, and 3 with $F=F_{1}^{A}=S \& P$ and $G=G_{1}^{A}=S S E . F_{1}^{A}$ and $G_{1}^{A}$ are defined in (2.1). 
Table 2: Results of the modified ASD test statistic for the Risk Averters

\begin{tabular}{|c|c|c|c|c|c|c|}
\hline \multirow{2}{*}{$\begin{array}{l}02 / 01 / 1996-19 / 05 / 2011 \\
F=\text { S\&P } 500, G=\text { SSE }\end{array}$} & \multicolumn{2}{|c|}{ FASD } & \multicolumn{2}{|c|}{ SASD } & \multicolumn{2}{|c|}{ 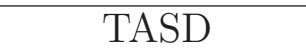 } \\
\hline & $T 1>0$ & $T 1<0$ & $T 2>0$ & $T 2<0$ & $T 3>0$ & $T 3<0$ \\
\hline $\operatorname{Total}(\%)$ & 34.92 & 27.92 & 0 & 89.57 & 0 & 98.98 \\
\hline Positive Domain(\%) & 34.92 & 0 & 0 & 0 & 0 & 0 \\
\hline Negative Domain(\%) & 0 & 27.92 & 0 & 89.57 & 0 & 98.98 \\
\hline $\max \left(\left|T_{j}^{A}\right|\right)$ & 9.10 & 7.36 & 0.68 & 7.16 & 1.00 & 6.20 \\
\hline 02/01/1996-09/03/2000 & \multicolumn{2}{|c|}{ FASD } & \multicolumn{2}{|c|}{ SASD } & \multicolumn{2}{|c|}{ TASD } \\
\hline$F=\mathrm{S} \& \mathrm{P} 500, G=\mathrm{SSE}$ & $T 1>0$ & $T 1<0$ & $T 2>0$ & $T 2<0$ & $T 3>0$ & $T 3<0$ \\
\hline $\operatorname{Total}(\%)$ & 36.91 & 31.59 & 0 & 91.29 & 0 & 99.32 \\
\hline Positive Domain(\%) & 36.91 & 0 & 0 & 0 & 0 & 0 \\
\hline Negative Domain $(\%)$ & 0 & 31.59 & 0 & 91.29 & 0 & 99.32 \\
\hline $\max \left(\left|T_{j}^{A}\right|\right)$ & 8.35 & 5.94 & 0.54 & 6.35 & 1.00 & 5.46 \\
\hline 09/03/2000-07/10/2002 & \multicolumn{2}{|c|}{ FASD } & \multicolumn{2}{|c|}{ SASD } & \multicolumn{2}{|c|}{ TASD } \\
\hline$F=\mathrm{S} \& \mathrm{P} 500, G=\mathrm{SSE}$ & $T 1>0$ & $T 1<0$ & $T 2>0$ & $T 2<0$ & $T 3>0$ & $T 3<0$ \\
\hline Total $(\%)$ & 38.82 & 4.02 & 63.98 & 0 & 20.21 & 0 \\
\hline Positive Domain(\%) & 38.82 & 0 & 63.98 & 0 & 20.21 & 0 \\
\hline Negative Domain(\%) & 0 & 4.02 & 0 & 0 & 0 & 0 \\
\hline $\max \left(\left|T_{j}^{A}\right|\right)$ & 4.83 & 3.59 & 3.52 & 1.00 & 2.14 & 1.00 \\
\hline 07/10/2002-16/10/2007 & \multicolumn{2}{|c|}{ FASD } & \multicolumn{2}{|c|}{$\overline{\mathrm{SASD}}$} & \multicolumn{2}{|c|}{ TASD } \\
\hline$F=\mathrm{S} \& \mathrm{P} 500, G=\mathrm{SSE}$ & $T 1>0$ & $T 1<0$ & $T 2>0$ & $T 2<0$ & $T 3>0$ & $T 3<0$ \\
\hline $\operatorname{Total}(\%)$ & 30.34 & 23.81 & 0 & 87.25 & 0 & 98.98 \\
\hline Positive Domain(\%) & 30.34 & 0 & 0 & 0 & 0 & 0 \\
\hline Negative Domain $(\%)$ & 0 & 23.81 & 0 & 87.25 & 0 & 98.98 \\
\hline $\max \left(\left|T_{j}^{A}\right|\right)$ & 8.86 & 7.48 & 1.04 & 6.84 & 0.74 & 5.42 \\
\hline /16/10/2007-04/11/2008 & \multicolumn{2}{|c|}{ FASD } & \multicolumn{2}{|c|}{ SASD } & \multicolumn{2}{|c|}{ TASD } \\
\hline$F=\mathrm{S} \& \mathrm{P} 500, G=\mathrm{SSE}$ & $T 1>0$ & $T 1<0$ & $T 2>0$ & $T 2<0$ & $T 3>0$ & $T 3<0$ \\
\hline $\operatorname{Total}(\%)$ & 0 & 25.28 & 0 & 78.86 & 0 & 61.13 \\
\hline Positive Domain(\%) & 0 & 0 & 0 & 0 & 0 & 0 \\
\hline Negative Domain $(\%)$ & 0 & 25.28 & 0 & 78.86 & 0 & 61.13 \\
\hline $\max \left(\left|T_{j}^{A}\right|\right)$ & 2.79 & 4.39 & 1.41 & 3.49 & 1.39 & 2.74 \\
\hline 04/11/2008-19/05/2011 & \multicolumn{2}{|c|}{ FASD } & \multicolumn{2}{|c|}{ SASD } & \multicolumn{2}{|c|}{ "TASD } \\
\hline$F=\mathrm{S} \& \mathrm{P} 500, G=\mathrm{SSE}$ & $T 1>0$ & $T 1<0$ & $T 2>0$ & $T 2<0$ & $T 3>0$ & $T 3<0$ \\
\hline $\operatorname{Total}(\%)$ & 15.94 & 0 & 0 & 0 & 0 & 0 \\
\hline Positive Domain(\%) & 15.94 & 0 & 0 & 0 & 0 & 0 \\
\hline Negative Domain(\%) & 0 & 0 & 0 & 0 & 0 & 0 \\
\hline $\max \left(\left|T_{j}^{A}\right|\right)$ & 3.73 & 2.41 & 1.00 & 1.14 & 1.00 & 0.56 \\
\hline
\end{tabular}

Note: This table summarizes the modified ASD test results for risk averters. The table reports the percentage of modified ASD statistics that are significantly negative or positive at the $5 \%$ significance level, based on the critical value generated from a bootstrap method discussed in Section 4. $T j$ is the test statistic $T_{j}^{A}(x)$ defined in (3.2) for $j=1,2$, and 3 with $F=S \& P$ and $G=S S E$. FASD, SASD, and TASD stand for first-, second-, and third-order ASD, respectively. 
Table 3: Results of the modified DSD test statistic for the Risk Seekers

\begin{tabular}{|c|c|c|c|c|c|c|}
\hline \multirow{2}{*}{$\begin{array}{l}02 / 01 / 1996-19 / 05 / 2011 \\
F=\mathrm{S} \& \mathrm{P} 500, G=\mathrm{SSE}\end{array}$} & \multicolumn{2}{|c|}{ 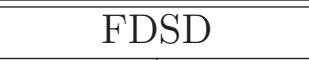 } & \multicolumn{2}{|c|}{ SDSD } & \multicolumn{2}{|c|}{ TDSD } \\
\hline & $T 1>0$ & $T 1<0$ & $T 2>0$ & $T 2<0$ & $T 3>0$ & $T 3<0$ \\
\hline $\operatorname{Total}(\%)$ & 27.92 & 34.92 & 0 & 96.42 & 0 & 98.96 \\
\hline Positive Domain(\%) & 27.92 & 0 & 0 & 0 & 0 & 0 \\
\hline Negative Domain $(\%)$ & 0 & 34.92 & 0 & 96.42 & 0 & 98.96 \\
\hline $\max \left(\left|T_{j}^{A}\right|\right)$ & 7.36 & 9.10 & 1.35 & 8.72 & 1.24 & 6.92 \\
\hline \multirow{2}{*}{$\begin{array}{l}02 / 01 / 1996-09 / 03 / 2000 \\
F=\mathrm{S} \& \mathrm{P} 500, G=\mathrm{SSE}\end{array}$} & \multicolumn{2}{|c|}{ FDSD } & \multicolumn{2}{|c|}{ SDSD } & \multicolumn{2}{|c|}{ TDSD } \\
\hline & $T 1>0$ & $T 1<0$ & $T 2>0$ & $T 2<0$ & $T 3>0$ & $T 3<0$ \\
\hline $\operatorname{Total}(\%)$ & 31.59 & 36.91 & 0 & 96.95 & 0 & 99.46 \\
\hline Positive Domain( $(\%)$ & 31.59 & 0 & 0 & 0 & 0 & 0 \\
\hline Negative Domain(\%) & 0 & 36.91 & 0 & 96.95 & 0 & 99.46 \\
\hline $\max \left(\left|T_{j}^{A}\right|\right)$ & 5.94 & 8.35 & 0.54 & 8.06 & 1.00 & 6.96 \\
\hline \multirow{2}{*}{$\begin{array}{l}\text { 09/03/2000-07/10/2002 } \\
F=\mathrm{S} \& \mathrm{P} 500, G=\mathrm{SSE}\end{array}$} & \multicolumn{2}{|c|}{ FDSD } & \multicolumn{2}{|c|}{ SDSD } & \multicolumn{2}{|c|}{ TDSD } \\
\hline & $T 1>0$ & $T 1<0$ & $T 2>0$ & $T 2<0$ & $T 3>0$ & $T 3<0$ \\
\hline $\operatorname{Total}(\%)$ & 4.02 & 43.38 & 0 & 0 & 0 & 0 \\
\hline Positive Domain(\%) & 4.02 & 0 & 0 & 0 & 0 & 0 \\
\hline Negative Domain(\%) & 0 & 43.38 & 0 & 0 & 0 & 0 \\
\hline $\max \left(\left|T_{j}^{A}\right|\right)$ & 3.59 & 4.83 & 2.23 & 1.63 & 0.73 & 1.51 \\
\hline \multirow{2}{*}{$\begin{array}{l}07 / 10 / 2002-16 / 10 / 2007 \\
F=\mathrm{S} \& \mathrm{P} 500, G=\mathrm{SSE}\end{array}$} & \multicolumn{2}{|c|}{ 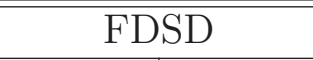 } & \multicolumn{2}{|c|}{ SDSD } & \multicolumn{2}{|c|}{ "TDSD } \\
\hline & $T 1>0$ & $T 1<0$ & $T 2>0$ & $T 2<0$ & $T 3>0$ & $T 3<0$ \\
\hline $\operatorname{Total}(\%)$ & 23.81 & 30.34 & 0 & 96.30 & 0 & 98.36 \\
\hline Positive Domain(\%) & 23.81 & 0 & 0 & 0 & 0 & 0 \\
\hline Negative Domain $(\%)$ & 0 & 30.34 & 0 & 96.30 & 0 & 98.36 \\
\hline $\max \left(\left|T_{j}^{A}\right|\right)$ & 7.48 & 8.86 & 1.00 & 8.64 & 1.00 & 7.32 \\
\hline \multirow{2}{*}{$\begin{array}{l}16 / 10 / 2007-04 / 11 / 2008 \\
F=\mathrm{S} \& \mathrm{P} 500, G=\mathrm{SSE}\end{array}$} & \multicolumn{2}{|c|}{ FDSD } & \multicolumn{2}{|c|}{ SDSD } & \multicolumn{2}{|c|}{ TDSD } \\
\hline & $T 1>0$ & $T 1<0$ & $T 2>0$ & $T 2<0$ & $T 3>0$ & $T 3<0$ \\
\hline $\operatorname{Total}(\%)$ & 25.28 & 0 & 0 & 0 & 0 & 0 \\
\hline Positive Domain(\%) & 25.28 & 0 & 0 & 0 & 0 & 0 \\
\hline Negative Domain(\%) & 0 & 0 & 0 & 0 & 0 & 0 \\
\hline $\max \left(\left|T_{j}^{A}\right|\right)$ & 4.39 & 2.79 & 1.53 & 1.75 & 1.31 & 1.09 \\
\hline \multirow{2}{*}{$\begin{array}{l}04 / 11 / 2008-19 / 05 / 2011 \\
F=\mathrm{S} \& \mathrm{P} 500, G=\mathrm{SSE}\end{array}$} & \multicolumn{2}{|c|}{ FDSD } & \multicolumn{2}{|c|}{ SDSD } & \multicolumn{2}{|c|}{ TDSD } \\
\hline & $T 1>0$ & $T 1<0$ & $T 2>0$ & $T 2<0$ & $T 3>0$ & $T 3<0$ \\
\hline Total $(\%)$ & 0 & 15.94 & 0 & 0 & 0 & 0 \\
\hline Positive Domain(\%) & 0 & 0 & 0 & 0 & 0 & 0 \\
\hline Negative Domain(\%) & 0 & 15.94 & 0 & 0 & 0 & 0 \\
\hline $\max \left(\left|T_{j}^{A}\right|\right)$ & 2.41 & 3.73 & 0.81 & 2.01 & 0.74 & 1.00 \\
\hline
\end{tabular}

Note: This table summarizes the modified DSD test results for risk seekers. The table reports the percentage of modified DSD statistics that are significantly negative or positive at the $5 \%$ significance level, based on the critical value generated from a bootstrap method discussed in Section 4. $T j$ is the test statistic $T_{j}^{D}(x)$ defined in (3.5) for $j=1,2$, and 3 with $F=S \& P$ and $G=S S E$. FDSD, SDSD, and TDSD stand for first-, second-, and third-order DSD, respectively. 
Table 4: Results of ASD and DSD Tests for the Risk Averters and Risk Seekers

\begin{tabular}{|c|c|c|}
\hline $01 / 1996-05 / 2011$ & $\mathrm{G}=\mathrm{S} \& \mathrm{P} 500$ & $\mathrm{SSE}$ \\
\hline $\mathrm{F}=\mathrm{S} \& \mathrm{P} 500$ & & $\mathrm{SASD}$ \\
$\mathrm{SSE}$ & $\mathrm{SDSD}$ & \\
\hline \hline $01 / 1996-03 / 2000$ & $\mathrm{G}=\mathrm{S} \& \mathrm{P} 500$ & $\mathrm{SSE}$ \\
\hline $\begin{array}{c}\mathrm{F}=\mathrm{S} \& \mathrm{P} 500 \\
\mathrm{SSE}\end{array}$ & $\mathrm{SDSD}$ & $\mathrm{SASD}$ \\
\hline \hline $03 / 2000-10 / 2002$ & $\mathrm{G}=\mathrm{S} \& \mathrm{P} 500$ & $\mathrm{SSE}$ \\
\hline $\mathrm{F}=\mathrm{S} \& \mathrm{P} 500$ & & $\mathrm{FASD} / \mathrm{FDSD} \#$ \\
$\mathrm{SSE}$ & & \\
\hline \hline $10 / 2002-10 / 2007$ & $\mathrm{G}=\mathrm{S} \& \mathrm{P} 500$ & $\mathrm{SSE}$ \\
\hline $\mathrm{F}=\mathrm{S} \& \mathrm{P} 500$ & & $\mathrm{SASD}$ \\
$\mathrm{SSE}$ & $\mathrm{SDSD}$ & \\
\hline \hline $10 / 2007-11 / 2008$ & $\mathrm{G}=\mathrm{S} \& \mathrm{P} 500$ & $\mathrm{SSE}$ \\
\hline $\mathrm{F}=\mathrm{S} \& \mathrm{P} 500$ & & $\mathrm{FASD} / \mathrm{FDSD}$ \\
$\mathrm{SSE}$ & & \\
\hline \hline $11 / 2008-05 / 2011$ & $\mathrm{G}=\mathrm{S} \& \mathrm{P} 500$ & $\mathrm{SSE}$ \\
\hline $\mathrm{F}=\mathrm{S} \& \mathrm{P} 500$ & & \\
$\mathrm{SSE}$ & $\mathrm{FASD} / \mathrm{FDSD} *$ & \\
\hline
\end{tabular}

Note: Here all the "SD" results are "F" (in the first column) SD "G" (in the first row). * means SD marginally and \# means SD when taking the concept of almost SD, Readers may refer to the text for more details. 
Figure 3: The modified DSD test statistics and the DSD integrals $F_{1}^{D}$ and $G_{1}^{D}$ from 02/01/1996 to $19 / 05 / 2011$

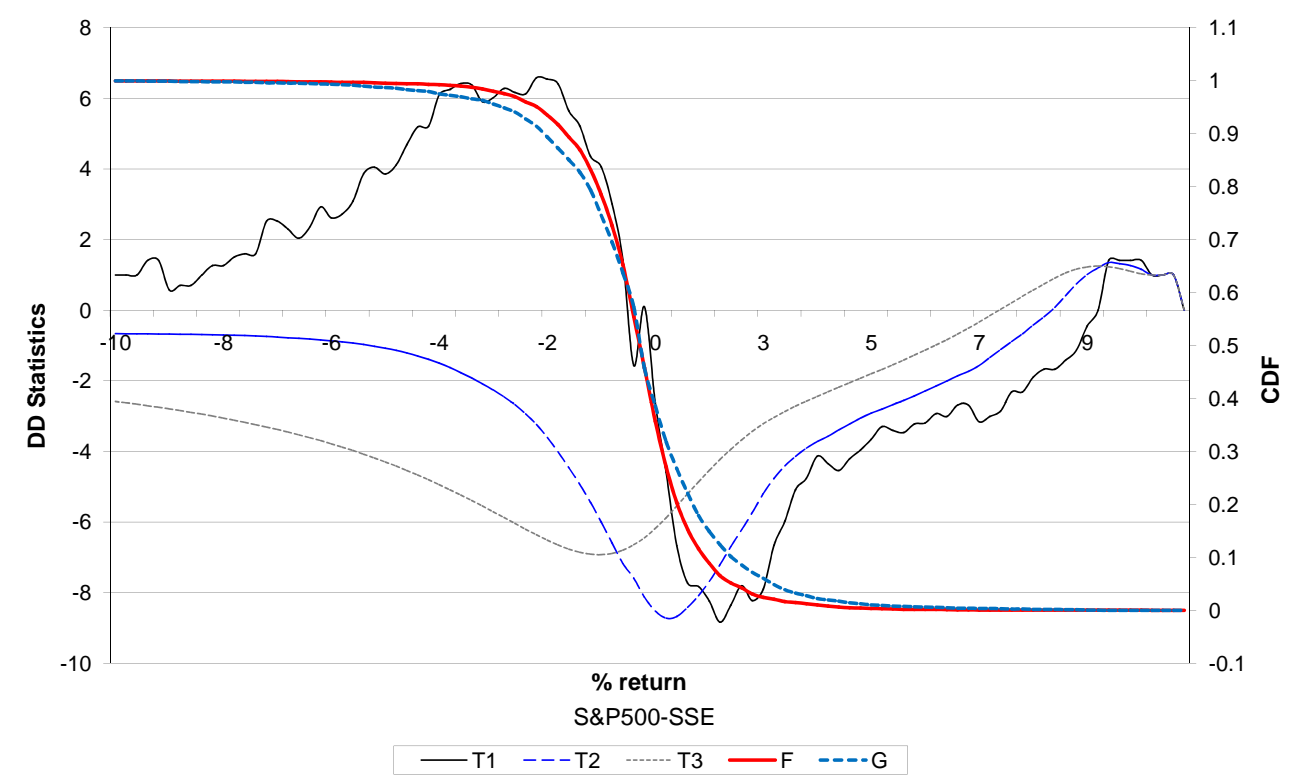

Note: $T j$ is the test statistic $T_{j}^{D}(x)$ defined in (3.5) for $j=1,2$, and 3 with $F=S \& P$ and $G=S S E$. $F_{1}^{D}$ and $G_{1}^{D}$ are defined in (2.1).

\section{References}

Anderson, G.J. 2004. Toward an Empirical Analysis of Polarization. Journal of Econometrics 122, 1-26.

Bai, Z.D., Hui, Y.C., Wong, W.K., Zitikis, R., 2012. Evaluating Prospect Performance: Making a Case for a Non-Asymptotic UMPU Test. Journal of Financial Econometrics, (forthcoming).

Bai, Z.D., Li, H., Liu, H.X., Wong, W.K., 2011. Test Statistics for Prospect and Markowitz Stochastic Dominances with Applications. Econometrics Journal 122, 1-26.

Bai, Z.D., H.X. Liu, W.K. Wong. 2009. Enhancement of the Applicability of Markowitz's Portfolio Optimization by Utilizing Random Matrix Theory. Mathematical Finance 19(4), 639-667.

Barrett, G., S. Donald. 2003. Consistent Tests for Stochastic Dominance. Econometrica $71,71-104$. 
Bishop, J.A., J.P. Formly, P.D. Thistle. 1992. Convergence of the South and non-South Income Distributions. American Economic Review 82, 262-272.

Broll, U., M. Egozcue, W.K. Wong, and R. Zitikis, (2010), Prospect Theory, Indifference Curves, and Hedging Risks, Applied Mathematics Research Express, 2010(2), 142-153. Broll, U., J.E. Wahl, and W.K. Wong, 2006, Elasticity of Risk Aversion and International Trade, Economics Letters 91(1), 126-130.

Chakravarty, S., Sarkar, A., Wu, L., 1998. Information asymmetry, market segmentation and the pricing of cross-listed shares: theory and evidence from Chinese A and B shares. Journal of International Financial Markets, Institutions and Money 8, 325356.

Chan, C.Y., de Peretti, C., Qiao, Z., Wong, W.K., 2012. Empirical Test of the Efficiency of UK Covered Warrants Market: Stochastic Dominance and Likelihood Ratio Test Approach. Journal of Empirical Finance 19(1), 162-174.

Davidson, R., J.Y Duclos. 2000. Statistical Inference for Stochastic Dominance and for the Measurement of Poverty and Inequality. Econometrica 68, 1435-1464.

Donsker, M.D. 1952. Justification and Extension of Doob's Heuristic Approach to the Kolmogorov-Smirnov Theorems. Annals of Mathematical Statistics 23, 277-281.

Egozcue, M., L. Fuentes García, W.K. Wong, and R. Zitikis, (2011): Do Investors Like to Diversify? A Study of Markowitz Preferences, European Journal of Operational Research 215(1), 188-193.

Egozcue, M., W.K. Wong. 2010. Gains from Diversification: A Majorization and Stochastic Dominance Approach. European Journal of Operational Research 200, 893-900.

Feldstein, M.S. 1969. Mean Variance Analysis in the Theory of Liquidity Preference and Portfolio Selection. Review of Economics Studies 36, 5-12.

Fong, W.M., H.H. Lean, and W.K. Wong, 2008, Stochastic Dominance and Behavior towards Risk: The Market for Internet Stocks, Journal of Economic Behavior and 
Organization 68(1), 194-208.

Fong, W.M., W.K. Wong, H.H. Lean. 2005. International Momentum Strategies: A Stochastic Dominance Approach. Journal of Financial Markets 8, 89-109.

Gasbarro, D., W.K. Wong, and J.K. Zumwalt, 2007, Stochastic dominance analysis of iShares, European Journal of Finance 13, 89-101.

Lam, K., T.S. Liu, W.K. Wong. 2010. A Pseudo-Bayesian Model in Financial Decision Making with Implications to Market Volatility, Under- and Overreaction. European Journal of Operational Research 203(1), 166-175.

Lam, K., T.S. Liu, W.K. Wong. 2012. A New Pseudo Bayesian Model with Implications to Financial Anomalies and Investors' Behaviors. Journal of Behavioral Finance (forthcoming).

Lean, H.H., McAleer, M., Wong, W.K., (2010), Market Efficiency of Oil Spot and Futures: A Mean-Variance and Stochastic Dominance Approach, Energy Economics 32, 979986.

Lean, H.H., W.K. Wong, X. Zhang. 2008. Size and Power of some Stochastic Dominance Tests. Journal of Statistical Computation and Simulation 79, 30-48.

Levy, H., H.M. Markowitz. 1979. Mean-Variance Approximations to Expected Utility. American Economic Review 69, 308-317.

Leung, P.L., W.K. Wong. 2008. On Testing the Equality of the Multiple Sharpe Ratios, with Application on the Evaluation of IShares. Journal of Risk 10(3), 1-16.

Li, C.K., W.K. Wong. 1999. Extension of Stochastic Dominance Theory to Random Variables. RAIRO - Operations Research 33(4), 509-524.

Linton, O., E. Maasoumi, Y.J. Whang. 2005. Consistent Testing for Stochastic Dominance under General Sampling Schemes. Review of Economic Studies 72, 735-765.

Ma, C. and W.K. Wong, (2010) Stochastic Dominance and Risk Measure: A DecisionTheoretic Foundation for VaR and C-VaR, European Journal of Operational Research 
207, 927-935.

Markowitz, H.M. 1952a. The Utility of Wealth. Journal of Political Economy 60, 151-156.

Markowitz, H.M. 1952b. Portfolio selection. Journal of Finance 7, 77-91.

Markowitz, H.M. 1959. Portfolio Selection: Efficient Diversification of Investments. New York: John Wiley and Sons.

Post, T., H. Levy. 2005. Does Risk Seeking Drive Asset Prices? A Stochastic Dominance Analysis of Aggregate Investor Preferences and Beliefs. Review of Financial Studies 18(3), 925-953.

Post, T., P. Versijp. 2007. Multivariate Tests for Stochastic Dominance Efficiency of a Given Portfolio. Journal of Financial and Quantitative Analysis 42(2), 489-516.

Richmond, J. 1982. A General Method for Constructing Simultaneous Confidence Intervals. Journal of the American Statistical Association 77, 455-460.

Schechtman, E., A. Shelef, S. Yitzhaki, and R. Zitikis. 2008. Testing Hypotheses about Absolute Concentration Curves and Marginal Conditional Stochastic Dominance. Econometric Theory 24: 1044-1062.

Sriboonchitta, S., W.K. Wong, S. Dhompongsa, H.T. Nguyen. 2009. Stochastic Dominance and Applications to Finance, Risk and Economics. Chapman and Hall/CRC, Taylor and Francis Group, Boca Raton, Florida, USA.

Stoline, M.R., H.K. Ury. 1979. Tables of the Studentized Maximum Modulus Distribution and an Application to Multiple Comparisons Among Means. Technometrics 21, 87-93. von Neumann, J., O. Morgenstern. 1944. Theory of Games and Economic Behavior. Princeton University Press, Princeton, NJ.

Wolfowitz, J. 1954. Generalization of the Theorem of Glivenko-Cantelli. Annals of Mathematical Statistics 25, 131-138.

Wong, W.K. 2007. Stochastic Dominance and Mean-Variance Measures of Profit and Loss for Business Planning and Investment. European Journal of Operational Research 182, 
829-843.

Wong, W.K., R. Chan. 2008. Markowitz and Prospect Stochastic Dominances. Annals of Finance 4(1), 105-129.

Wong, W.K., C.K. Li. 1999. A Note on Convex Stochastic Dominance Theory. Economics Letters 62, 293-300.

Wong, W.K., C. Ma. 2008. Preferences over Meyer's Location-Scale Family. Economic Theory 37(1), 119-146. 